\title{
Target Product Profile Analysis of COVID-19 Vaccines in Phase III Clinical Trials and Beyond: An Early 2021 Perspective
}

\author{
Colin D. Funk ${ }^{1,2, * \mathbb{C}}$, Craig Laferrière $^{2}$ and Ali Ardakani ${ }^{2} \mathbb{0}$ \\ 1 Department of Biomedical and Molecular Sciences, Queen's University, Kingston, ON K7L 3N6, Canada \\ 2 Scientific Research Division, Novateur Ventures Inc., Vancouver, BC V6E 3P3, Canada; \\ craiglaferriere@gmail.com (C.L.); ali@novateur.org (A.A.) \\ * Correspondence: funkc@queensu.ca
}

Citation: Funk, C.D.; Laferrière, C.; Ardakani, A. Target Product Profile Analysis of COVID-19 Vaccines in Phase III Clinical Trials and Beyond: An Early 2021 Perspective. Viruses 2021, 13, 418. https://doi.org/ $10.3390 / \mathrm{v} 13030418$

Academic Editor:

Kenneth Lundstrom

Received: 11 February 2021

Accepted: 3 March 2021

Published: 5 March 2021

Publisher's Note: MDPI stays neutral with regard to jurisdictional claims in published maps and institutional affiliations.

Copyright: (C) 2021 by the authors. Licensee MDPI, Basel, Switzerland. This article is an open access article distributed under the terms and conditions of the Creative Commons Attribution (CC BY) license (https:// creativecommons.org/licenses/by/ $4.0 /)$.

\begin{abstract}
The coronavirus SARS-CoV-2, which causes Coronavirus disease 2019 (COVID-19), has infected more than 100 million people globally and caused over 2.5 million deaths in just over one year since its discovery in Wuhan, China in December 2019. The pandemic has evoked widespread collateral damage to societies and economies, and has destabilized mental health and well-being. Early in 2020, unprecedented efforts went into the development of vaccines that generate effective antibodies to the SARS-CoV-2 virus. Teams developing twelve candidate vaccines, based on four platforms (messenger RNA, non-replicating viral vector, protein/virus-like particle, and inactivated virus) had initiated or announced the Phase III clinical trial stage by early November 2020, with several having received emergency use authorization in less than a year. Vaccine rollout has proceeded around the globe. Previously, we and others had proposed a target product profile (TPP) for ideal/optimal and acceptable/minimal COVID-19 vaccines. How well do these candidate vaccines stack up to a harmonized TPP? Here, we perform a comparative analysis in several categories of these candidate vaccines based on the latest available trial data and highlight the early successes as well as the hurdles and barriers yet to be overcome for ending the global COVID-19 pandemic.
\end{abstract}

Keywords: COVID-19; SARS-CoV-2; vaccine; target product profile; immune response; coronavirus; clinical trial; public health

\section{Introduction}

The pandemic of 2020 caused by the global dissemination of the SARS-CoV-2 coronavirus has crippled the world. Tens of millions of infections and over two million deaths (https: / / coronavirus.jhu.edu/map.html (accessed on 9 February 2021)) from coronavirus disease 2019 (COVID-19) have given rise to sorrow, social isolation, and disruption of life as we know it. An immense body of the literature has been accumulating in the past year explaining the epidemiology and infectious mechanisms of SARS-CoV-2, the pathogenesis of COVID-19, and the development of potential therapies and vaccines [1-14]. In June 2020, we had previously provided a global snapshot of the race for an approved vaccine targeting SARS-CoV-2 and outlined what the ideal vaccine should look like via a proposed target product profile (TPP) [15]. A TPP for a COVID-19 vaccine defines a preferred and minimally acceptable outline for generation of immunity to protect populations from the risk of disease and for reactive use in pandemic outbreaks. Currently, there are more than 200 vaccines at various stages of development specifically targeting SARS-CoV-2 according to the latest World Health Organization (WHO) database (https:/ / www.who. int/publications/m/item/draft-landscape-of-covid-19-candidate-vaccines (accessed on 9 February 2021)). Of these, twelve had reached/announced Phase III clinical trials by mid-November 2020, having initiated development months before this time. Several more have attained this stage in the ensuing months but we are limiting our analysis to these first twelve candidates. A comparative chart of guidelines for a COVID-19 vaccine TPP from four sources (ours [15], WHO (https:/ / www.who.int/publications/m/item/who-target- 
product-profiles-for-covid-19-vaccines (accessed on 9 February 2021)), Coalition for Epidemic Preparedness Innovations (CEPI) (https:/ / media.tghn.org/articles/TPC_COVID19_CEPI_Version_1.1_20200303.pdf (accessed on 9 February 2021)) and Center for Biologics Evaluations and Research (CBER) (https:/ / www.fda.gov/media/139638/download (accessed on 9 February 2021)) is presented in Table S1 of the Supplementary Materials.

Gavi, the Vaccine Alliance, with its associated COVAX vaccine pillar of Access to COVID-19 Tools (ACT) Accelerator (https://www.gavi.org/ (accessed on 9 February 2021); https:/ /www.gavi.org/covax-facility (accessed on 9 February 2021)), which includes CEPI and WHO partnerships, in addition to Operation Warp Speed (to be renamed) (https:/ / www.bloomberg.com/news/articles/2021-01-15/biden-team-is-retiringwarp-speed-name-from-vaccination-push (accessed on 9 February 2021)) and other company initiatives have paved the way for the rollout of vaccine candidates to the world's population. This includes safety testing, manufacturing, distribution and logistics for providing billions of doses to combat COVID-19 and end the global pandemic. Here, we update and revise the outlook of the most advanced candidate vaccines in five (of fourteen; see Table S1) categories (safety/reactogenicity, efficacy/immunogenicity, dosing regimen, product stability/storage/supply chain (i.e., logistics) and target price/accessibility) to see how they stack up to a harmonized TPP (see below).

\section{Overview of Twelve Vaccines Targeting COVID-19 in Phase III Clinical Trials}

Within ten months' time, twelve vaccine candidates had been officially registered into Phase III clinical trials (https:/ / www.who.int/publications/m/item/draft-landscape-ofcovid-19-candidate-vaccines (accessed on 9 February 2021)). This is a phenomenal and unprecedented feat in the history of medicine, owing to a worldwide concerted effort to eradicate this viral pandemic. The process of vaccine development normally takes place over several years but has now been accelerated through concurrent pre-clinical and early Phase I studies, strategic risk measures and adaptive trial designs. Here, we consider vaccine candidates, two each from the messenger RNA and protein/virus-like protein platforms and four each from the viral vector (non-replicating) and inactivated virus platforms (Figure 1). Each of these platforms, and 9 of 12 vaccine candidates were covered partially in our previous publication [15]. We will briefly review and update the salient characteristics of each here (Table 1).

\subsection{Messenger RNA ( $m R N A$ ) Vaccines}

The Moderna mRNA-1273 [22] and Pfizer/BioNtech BNT162b2 (also known by brand name Comirnaty or by generic name tozinameran) (https://www.fiercepharma.com/ marketing/pfizer-biontech-select-comirnaty-as-brand-name-for-covid-19-vaccine (accessed on 9 February 2021)) [23] mRNA vaccines have been the frontrunners in the vaccine "race" since early 2020 when the pandemic was first declared [15]. Although an mRNA vaccine has never before been approved by regulatory agencies, this has now changed with the United States Food and Drug Administration (FDA) emergency use authorization (EUA) of these two candidates [24] in December 2020, after an astoundingly short 11 month journey, a process that normally takes place over 10+ years. Both vaccines employ an mRNA, which targets the pre-fusion stabilized SARS-CoV-2 full-length spike P2-mutant version, as the immunogen, wrapped in proprietary lipid nanoparticle (LNP) formulations (see [24] and Supplementary Materials Table S2 for the precise compositions of the LNPs) in a prime-boost format (Pfizer/BioNTech: $30 \mu \mathrm{g}$ immunogen, 21 days apart and Moderna $100 \mu \mathrm{g}$ immunogen, 28 days apart). Both have documented $94-95 \%$ efficacy based on 30-44,000 participants in their respective Phase III double-blinded, randomized placebocontrolled studies ([16,17]; see Figure 1, Table 1). These remarkably positive findings overwhelmingly exceeded early expectations. 


\section{Vaccines in Phase III (and beyond)}

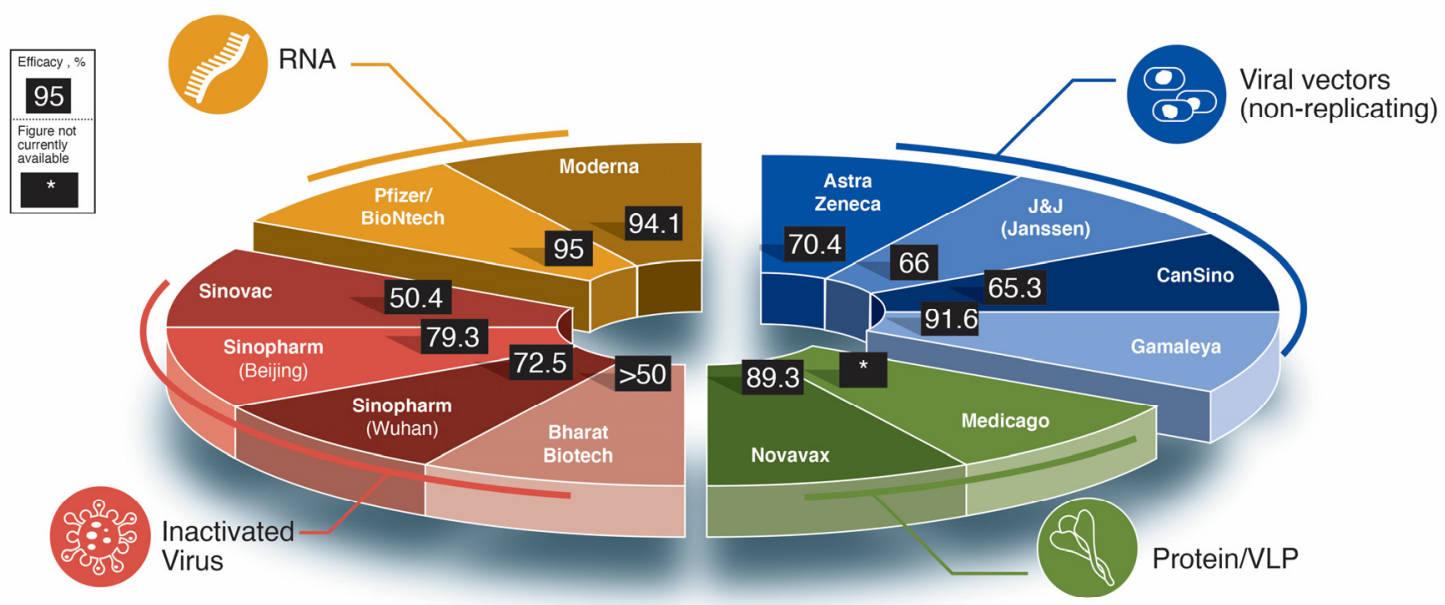

Figure 1. The twelve SARS-CoV-2 vaccine candidates reaching/announcing Phase III clinical trials by mid-November 2020. Shown in pie-chart configuration are the companies responsible for the development of the vaccines as well as their reported efficacy in Phase III trials. *, efficacy not yet available. Due to variability in reporting criteria for cases of COVID-19, efficacy results may not be directly comparable.

\subsection{Viral Vector-Based (Non-Replicating) Vaccines}

Four groups (Astra Zeneca/University of Oxford (AZ/Ox), CanSino Biologics, Gamaleya Research Institute and Johnson \& Johnson/Janssen (J\&J)) have all adopted the use of non-replicating adenoviral vectors to drive the expression of the full-length SARS-CoV-2 spike glycoprotein $\left(5 \times 10^{10}\right.$ or $10^{11}$ viral particles for each dose injection) to induce an immune response $[18,19,25-31]$. The differences between them lie in the chosen vector strain and immunization schedules. All except J\&J use a prime-boost scheme. AZ/Ox (vaccine named AZ1222 or Covishield in India) (https://www.bbc.com/news/worldasia-india-55748124 (accessed on 9 February 2021)) uses an adenoviral vector that infects chimpanzees but not humans $[25,26]$. CanSino $(\mathrm{Ad} 5-\mathrm{nCoV})$ uses a recombinant version of Ad5 adenovirus that naturally transmits in humans [28,29], while J\&J (Ad26.COV2.S) uses a variant adenovirus Ad26 that is not normally (or only rarely) encountered by the human population [31]. Gamaleya (Sputnik V) uses a combination prime with Ad26 and boost with Ad5 [19,30]. From 12-40,000 have been enrolled in Phase III trials for each of the four members of this platform. Efficacy results have ranged from promising $(91.6 \%$ for Sputnik V) to variable (62-90\% for AZ/Ox where a clinical trial "mistake" led to a surprising increased efficacy) $[18,32,33]$ (see Figure 1, Table 1). 
Table 1. Summary of Vaccines Analyzed in this Study.

\begin{tabular}{|c|c|c|c|c|c|c|c|}
\hline $\begin{array}{l}\text { Platform } \\
\text { [Ref.] }\end{array}$ & Name (Dose) & $\begin{array}{c}\text { Phase III } \\
\text { \# Enrolled Placebo }\end{array}$ & $\begin{array}{c}\text { Phase III } \\
\text { \# Enrolled Vaccine }\end{array}$ & $\begin{array}{l}\text { COVID Cases } \\
\text { Placebo (Severe) }\end{array}$ & $\begin{array}{l}\text { COVID Cases } \\
\text { Vaccine (Severe) }\end{array}$ & $\begin{array}{l}\text { \% Efficacy } \\
\text { (Severe) }\end{array}$ & $\begin{array}{c}\text { Projected } \\
\text { Cost/Dose (USD) }\end{array}$ \\
\hline \multicolumn{8}{|l|}{ RNA } \\
\hline $\begin{array}{c}\text { Pfizer/BioNTech } \\
\text { [16] }\end{array}$ & $\begin{array}{c}\text { BNT162b2 } \\
(2 \times 30 \mu \mathrm{g}, 21 \text { days apart })\end{array}$ & 21,720 & 21,728 & $\begin{array}{l}162 \\
(9)\end{array}$ & $\begin{array}{c}8 \\
(1)\end{array}$ & $\begin{array}{c}95 \\
(89)\end{array}$ & $\$ 14.70-19.50$ \\
\hline $\begin{array}{l}\text { Moderna } \\
{[17]}\end{array}$ & mRNA-1273 $(2 \times 100$ g, 28 days apart $)$ & 15,210 & 15,210 & $\begin{array}{l}185 \\
(30)\end{array}$ & $\begin{array}{l}11 \\
(0)\end{array}$ & $\begin{array}{c}94.1 \\
(100)\end{array}$ & $\$ 25-37$ \\
\hline \multicolumn{8}{|l|}{ Viral Vector } \\
\hline $\begin{array}{c}\text { Astra Zeneca } \\
\text { [18] }\end{array}$ & $\begin{array}{l}\text { AZD1222 }\left(2 \times 5 \times 10^{10} \mathrm{vp}, 28 \text { days apart }\right) \\
\left(2.2 \times 10^{10} \mathrm{vp} \text {, then } 5 \times 10^{10} 28 \text { days apart }\right)\end{array}$ & $\begin{array}{c}4455 \\
{[1374]}\end{array}$ & $\begin{array}{c}4440 \\
{[1367]}\end{array}$ & $\begin{array}{c}71 \\
{[30]} \\
(10 / 2) \\
\end{array}$ & $\begin{array}{c}27 \\
{[3]} \\
(0 / 0)\end{array}$ & $\begin{array}{l}62.1(70.4) \\
{[90]} \\
(100)\end{array}$ & $\$ 2-10^{+}$ \\
\hline CanSino $^{b}$ & $\begin{array}{c}\text { Ad5-nCoV (Convidecia) (single dose } 5 \times 10^{10} \\
\text { vp) }\end{array}$ & \multicolumn{2}{|c|}{ 30,000 (Pakistan) } & - & - & $\begin{array}{c}65.37 \\
(91)\end{array}$ & $\$<4$ \\
\hline Gamaleya $^{c}$ & $\begin{array}{l}\text { Sputnik V (Gam-COVID-Vac) }\left(10^{11} \mathrm{vp}\right. \\
\text { rAd26-S, followed by } 10^{11} \text { vp rAd5-S } \\
21 \text { days apart })\end{array}$ & 5476 & 16,501 & 62 & 16 & 91.6 & $\$<10$ \\
\hline \multicolumn{8}{|l|}{ Protein/VLP } \\
\hline Novavax ${ }^{e}$ & $\begin{array}{l}\text { NVX-CoV2373 }(2 \times 5 \mu \mathrm{g}+50 \mu \mathrm{g} \mathrm{M} 1 \\
\text { adjuvant, } 21 \text { days apart })\end{array}$ & \multicolumn{2}{|c|}{$15,000^{+}(\mathrm{UK})$} & $\begin{array}{l}56 \\
(1)\end{array}$ & $\begin{array}{c}6 \\
(0)\end{array}$ & 89.3 (UK) & $\$ 16$ \\
\hline Medicago ${ }^{f}$ & $\begin{array}{c}\text { CoVLP }(2 \times 3.75 \mu \mathrm{g}+\text { AS03 GSK adjuvant, } \\
21 \text { days apart })\end{array}$ & \multicolumn{2}{|c|}{30,000} & - & - & - & Not known \\
\hline \multicolumn{8}{|l|}{ Inactivated } \\
\hline Sinovac $\mathrm{g}$ & $\begin{array}{c}\text { CoronaVac }(2 \times \text { alum-adjuvanted } 14 \text { days } \\
\text { apart })\end{array}$ & \multicolumn{2}{|c|}{9200 (Brazil) } & $\begin{array}{l}167 \\
(31)\end{array}$ & $\begin{array}{l}85 \\
(7)\end{array}$ & 50.4 (Brazil) & $\$ 30$ \\
\hline $\begin{array}{l}\text { Sinopharm } \\
\text { (Beijing) }\end{array}$ & $\begin{array}{c}\text { BBIBP-CorV }(2 \times 4 \mu \mathrm{g} \text { in alum adjuvant } \\
21 \text { days apart })\end{array}$ & \multicolumn{2}{|c|}{ 10,000 (UAE) } & - & - & 79.3 (UAE) & $\$ 30-72.50$ \\
\hline $\begin{array}{l}\text { Sinopharm }{ }^{\mathrm{i}} \\
\text { (Wuhan) }\end{array}$ & $\begin{array}{c}\text { Vero }(2 \times 5 \mu \mathrm{g} \text { in alum adjuvant } \\
21 \text { days apart })\end{array}$ & \multicolumn{2}{|c|}{$\begin{array}{l}\text { 27,000 (Peru, UAE, Bahrain, Morocco, } \\
\text { Argentina, Jordan and Pakistan) }\end{array}$} & - & - & $72.5 \%$ & $\$ 30-72.50$ \\
\hline
\end{tabular}


Table 1. Cont.

\begin{tabular}{|c|c|c|c|c|c|c|c|}
\hline $\begin{array}{l}\text { Platform } \\
\text { [Ref.] }\end{array}$ & Name (Dose) & $\begin{array}{c}\text { Phase III } \\
\text { \# Enrolled Placebo }\end{array}$ & $\begin{array}{l}\text { Phase III } \\
\text { \# Enrolled Vaccine }\end{array}$ & $\begin{array}{l}\text { COVID Cases } \\
\text { Placebo (Severe) }\end{array}$ & $\begin{array}{l}\text { COVID Cases } \\
\text { Vaccine (Severe) }\end{array}$ & $\begin{array}{l}\text { \% Efficacy } \\
\text { (Severe) }\end{array}$ & $\begin{array}{c}\text { Projected } \\
\text { Cost/Dose (USD) }\end{array}$ \\
\hline Bharat Biotech ${ }^{j}$ & $\begin{array}{l}\text { COVAXIN (BBV152) }(2 \times 6 \mu \mathrm{g} \text { in } \\
\text { Alhydroxiquim-II adjuvant } 28 \text { days apart })\end{array}$ & \multicolumn{2}{|c|}{25,800} & - & - & $>50 \%$ & $\$ 2-3$ \\
\hline
\end{tabular}

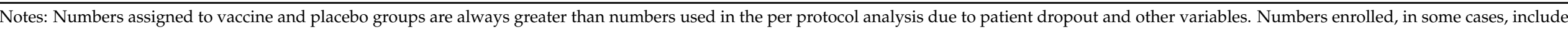

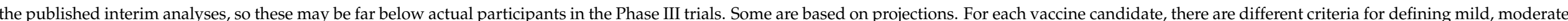

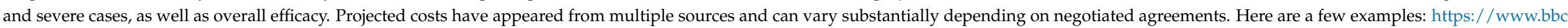

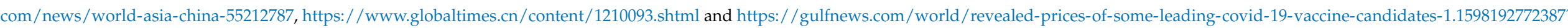

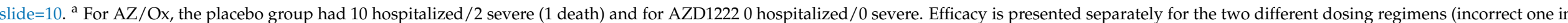

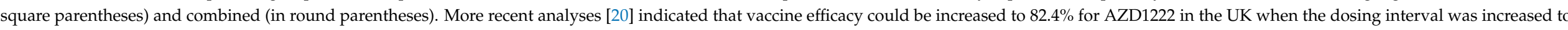

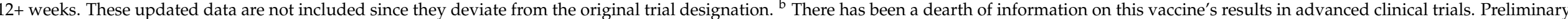

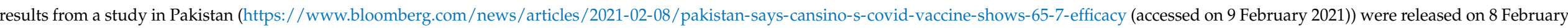

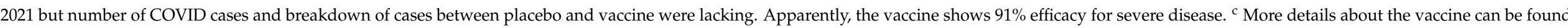

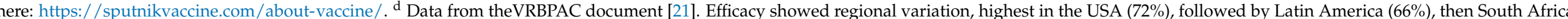

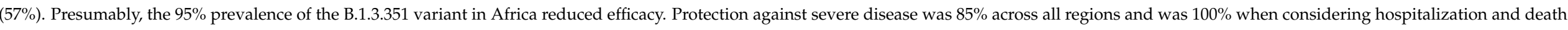

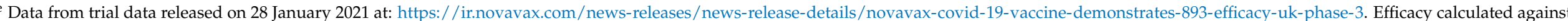

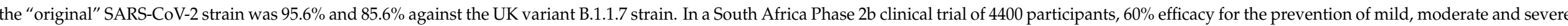

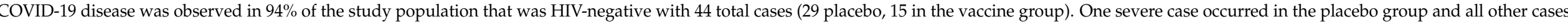

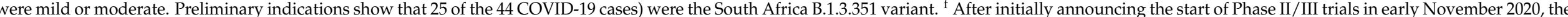

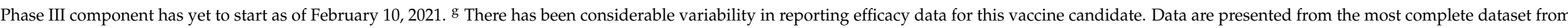

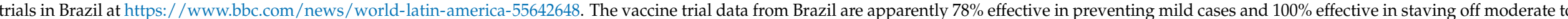

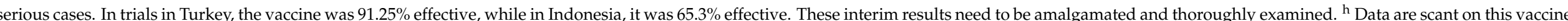

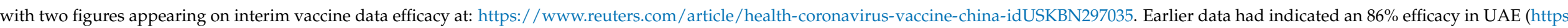

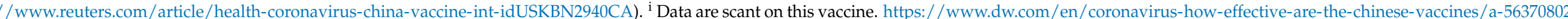

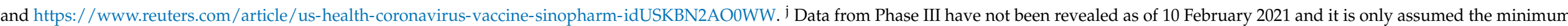

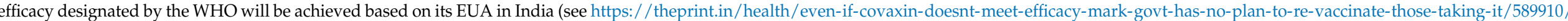
and https://indianexpress.com/article/explained/coronavirus-vaccines-india-covishield-bharat-biotech-covaxin-7131057/). 


\subsection{Recombinant Protein-Based Vaccines}

The protein subunit Novavax candidate NVX-CoV2373 uses the recombinant, properly folded, full-length SARS-CoV-2 spike glycoprotein in the pre-fusion state engineered from insect cells in a nanoparticle formulation along with their proprietary saponinbased Matrix-M adjuvant [34]. Medicago also utilizes the full-length spike glycoprotein with several key changes: (i) a plant gene signal peptide; (ii) three stabilizing substitutions at the S1/S2 cleavage site; (iii) the dual proline (P2) prefusion conformationstabilizing exchanges; and (iv) transmembrane and cytoplasmic tail sections swapped with influenza sequences) expressed in tobacco (Nicotiana benthamiana) plants [35]. Swapping the influenza domains increases viral-like protein (VLP) assembly and budding. Medicago utilizes the AS03 adjuvant, an oil-in-water emulsion containing tocopherol and squalene supplied by GlaxoSmithKline, which is mixed immediately prior to administration. Both use a prime-boost regimen 21 days apart with either 5 (Novavax) or 3.75 (Medicago) $\mu \mathrm{g}$ protein/dose. Early indications show excellent efficacy (just below 90\%) (https:/ /ir.novavax.com/news-releases/news-release-details/novavax-covid19-vaccine-demonstrates-893-efficacy-uk-phase-3 (accessed on 9 February 2021)) for NVXCoV2373.

\subsection{Inactivated Virus}

Three Chinese conglomerates and one Indian company have used the tried-and-true method of viral inactivation by $\beta$-propriolactone treatment, along with various alum adjuvants for generation of their SARS-CoV-2 vaccine candidates [36-42]. Each is using the prime-boost regimen with 4-6 $\mu \mathrm{g}$ virus and 14-28 days separation between prime and booster injections.

\section{Clinical Trial Data of Twelve Vaccines Targeting COVID-19 in Phase III Trials and Emergency Use Authorization}

3.1. mRNA Vaccines—Safety/Reactogenicity and Immunogenicity/Efficacy

The Phase III data for both Pfizer/BioNTech and Moderna vaccines that are built on the same platform, with nearly identical antigen-producing mRNA sequences and their own unique blend of LNP encapsulation, yielded remarkably similar results in terms of safety and efficacy $[16,17,22,43,44]$. The most common solicited local and systemic reactogenicity were pain at the injection site, headache, and fever (defined as temperature $\geq 38^{\circ} \mathrm{C}$ ). In most cases, these adverse events (AEs) were reported for 7 days post-injection (Table 2). In general, AEs were more abundant after the boosting dose. Table 2 shows that mRNA vaccines induced fever in $0.8-3 \%$ of subjects after the first dose. The mRNA vaccines have a reactogenicity profile similar to other vaccines commonly used in adults. For example, the pneumonia vaccine Prevnar ${ }^{\circledR} 13$ induced fever in the range 1.0-7.2\% of subjects (Prevnar Product Monograph [45]). The shingles vaccine Shingrix induced fever $\left(>37.5^{\circ} \mathrm{C}\right)$ in $14.3-25.9 \%$ of adult subjects aged 50 years and above (Shingrix Product Monograph [46]). The HPV vaccine Gardasil ${ }^{\circledR}$ induced fever $\left(>37.8^{\circ} \mathrm{C}\right)$ in $2.5 \%$ of women aged 27 to 45 years (Gardasil-9 Product Monograph [47]). Influenza vaccine tends to induce less fever, around 1-2\% for adults over 18 years of age (Fluviral product monograph [48]).

Efficacy for both mRNA vaccine candidates was nearly identical at 94-95\% $[16,17]$. These results represent a resounding success for the novel platform technology, which has never previously received approval for any vaccine or drug program. Since receiving emergency use authorization (EUA) approval, severe allergic reaction cases have arisen during vaccine rollout $[50,51]$, which required emergency epinephrine rescue [52]. The case numbers exceed the typical rare " 1 in a million" side effects that emerge in the general population after clinical trials, where exclusion criteria limit involvement of allergic individuals and other potentially vulnerable participants. Early speculation centers around an adverse anaphylactoid reaction to the polyethylene glycol (PEG) that is found in both formulations [52,53]. The mechanism of action is not understood, but hypotheses include IgE antibodies to PEG, non-IgE complement activation-related pseudo-allergy (CARPA), 
nanoparticle aggregation and a negatively charged linkage of lipid to PEG [52,53]. There appears to be a statistically significant difference in the rate of allergic reactions between the Pfizer and Moderna vaccines ( 11.1 [50] vs. 2.5 [51] per million injections, respectively; $p<0.001$, Fisher's exact) and a comparison of the Pfizer and Moderna lipid nanoparticle formulations presented in the Supplementary material Table S2 shows that the difference in the lipidated-PEG molecules between these two vaccines is at the linkage between the lipid and the PEG. The two vaccines also have different cationic lipids, SM-102 and ALC-0315, but these have not been implicated in the allergic reactions.

Table 2. Solicited reactogenicity 7 days post injection, all ages, Phase 3 or Phase $1 / 2$ data of the chosen final formulation and schedule.

\begin{tabular}{|c|c|c|c|c|c|c|c|c|}
\hline & \multirow[t]{2}{*}{ Dose } & \multirow[t]{2}{*}{$\mathbf{N}$} & \multicolumn{2}{|c|}{ Injection Site Pain } & \multicolumn{2}{|c|}{ Headache } & \multicolumn{2}{|c|}{ Fever } \\
\hline Grade & & & \multicolumn{2}{|c|}{ Any } & \multicolumn{2}{|c|}{ Any } & \multicolumn{2}{|c|}{ Oral $\geq 38^{\circ} \mathrm{C}$} \\
\hline \multicolumn{2}{|l|}{ Group } & $\begin{array}{c}\text { Placebo + } \\
\text { Vaccine }\end{array}$ & Placebo & Vaccine & Placebo & Vaccine & Placebo & Vaccine \\
\hline \multicolumn{9}{|l|}{ mRNA } \\
\hline \multirow{2}{*}{$\begin{array}{c}\text { Pfizer/BioNTech } \\
\text { BNT162b2 } \\
(2 \times 30 \mu \mathrm{g}, 21 \text { d.a. })[16,43]\end{array}$} & Post dose 1 & $\sim 8183^{\mathrm{a}}$ & $12 \%$ & $78 \%$ & $27 \%$ & $35 \%$ & $0.6 \%$ & $3 \%$ \\
\hline & Post dose 2 & $\sim 8183^{a}$ & $10 \%$ & $73 \%$ & $20 \%$ & $47 \%$ & $0 \%$ & $14 \%$ \\
\hline \multirow{2}{*}{$\begin{array}{c}\text { Moderna } \\
\text { mRNA-1273 }(2 \times 100 \mu \mathrm{g}, 28 \text { d.a. }) \\
{[17,22,44]}\end{array}$} & Post dose 1 & $\sim 30,300^{b}$ & $17.5 \%$ & $83.7 \%$ & $26.6 \%$ & $32.7 \%$ & $0.3 \%$ & $0.8 \%$ \\
\hline & Post dose 2 & $\sim 29,200^{b}$ & $17.0 \%$ & $88.2 \%$ & $23.4 \%$ & $58.6 \%$ & $0.3 \%$ & $15.5 \%$ \\
\hline \multicolumn{9}{|l|}{ Viral Vector } \\
\hline \multirow{2}{*}{$\begin{array}{c}\text { Astra Zeneca AZD1222 } \\
\left(2 \times 5 \times 10^{10} \mathrm{vp}, 28 \text { d.a. }\right)[18,25,26]\end{array}$} & Post dose 1 & $964^{c}$ & $37 \%$ & $67 \%$ & $41 \%$ & $68 \%$ & $2 \%$ & $18 \%$ \\
\hline & Post dose 2 & $128^{d}$ & - & $30.7 \%$ & - & $\%$ & - & $0 \%$ \\
\hline \multirow{2}{*}{$\begin{array}{c}\text { CanSino } \\
\left.\text { Ad5-nCoV (single dose } 5 \times 10^{10} \mathrm{vp}\right) \\
{[28,29]}\end{array}$} & Post dose 1 & $255^{e}$ & $9 \%$ & $56 \%$ & $13 \%$ & $28 \%$ & $10 \% \mathrm{f}$ & $16 \% \mathrm{f}$ \\
\hline & Post dose 2 & NA & NA & NA & NA & NA & NA & NA \\
\hline \multirow{2}{*}{$\begin{array}{c}\text { Gamaleya Sputnik V } \\
\left(10^{11} \text { vp Ad26 then } 10^{11} \text { Ad5 } 21 \text { d.a. }\right) \\
{[30]}\end{array}$} & Post dose 1 & $9^{g}$ & - & $78 \%$ & - & $67 \%$ & - & $89 \%$ h \\
\hline & Post dose 2 & $20 \mathrm{~g}$ & - & $40 \%$ & - & $55 \%$ & - & $100 \%^{h}$ \\
\hline \multirow{2}{*}{$\begin{array}{l}\text { J\&J (Janssen) }(\text { Ad26.COV2.S } \\
\text { single } 5 \times 10^{10} \text { vp) }[21,49]\end{array}$} & Post dose 1 & $673^{j}$ & $16.7 \%$ & $48.7 \%$ & $23.9 \%$ & $39.2 \%$ & $0.9 \%$ & $9.1 \%$ \\
\hline & Post dose 2 & NA & NA & NA & NA & NA & NA & NA \\
\hline \multicolumn{9}{|l|}{ Protein/VLP } \\
\hline \multirow{2}{*}{$\begin{array}{c}\text { Novavax } \\
\text { NVX-CoV2373 }(2 \times 5 \mu \mathrm{g}+50 \mu \mathrm{g} \mathrm{M1} \\
21 \text { d.a. })[34]\end{array}$} & Post dose 1 & $49^{k}$ & $13 \%$ & $38 \%$ & $31 \%$ & $23 \%$ & $0 \%$ & $0 \%$ \\
\hline & Post dose 2 & $49^{k}$ & $10 \%$ & $58 \%$ & $29 \%$ & $47 \%$ & $0 \%$ & $0 \%$ \\
\hline \multirow{2}{*}{$\begin{array}{c}\text { Medicago } \\
\text { CoVLP } 2 \times 3.75 \mu \mathrm{g}+\text { AS03, } 21 \text { d.a. [35] }\end{array}$} & Post dose 1 & $20^{\mathrm{m}}$ & - & $95 \%$ & - & $30 \%$ & - & $0 \%$ \\
\hline & Post dose 2 & $19^{\mathrm{m}}$ & - & $100 \%$ & - & $57.9 \%$ & - & $36.8 \%$ \\
\hline \multicolumn{9}{|l|}{ Inactivated } \\
\hline \multirow{2}{*}{$\begin{array}{c}\text { Sinovac CoronaVac }(2 \times 3 \mu \mathrm{g}+\text { alum } 14 \\
\text { d.a. })[36,37]\end{array}$} & Post dose 1 & $180^{\mathrm{p}}$ & $10 \%$ & $9.2 \%$ & $1.7 \%$ & $0.8 \%$ & $1.7 \%$ & $2.5 \%$ \\
\hline & Post dose 2 & $180^{\mathrm{P}}$ & $3.3 \%$ & $13.6 \%$ & $0 \%$ & $0.8 \%$ & $0 \%$ & $1.7 \%$ \\
\hline \multirow{2}{*}{$\begin{array}{c}\text { Sinopharm BBIBP-CorV }(2 \times 4 \mu \mathrm{g} v \mathrm{vp}+ \\
\text { alum, } 21 \text { d.a. })[38]\end{array}$} & $\begin{array}{l}\text { Post dose } 1 \\
\quad \text { or } 2 \mathrm{q}\end{array}$ & 112 & $4 \%$ & $12 \%$ & $7 \%$ & $1 \%$ & $4 \%$ & $4 \%$ \\
\hline & Post dose 2 & - & - & - & - & - & - & - \\
\hline \multirow{2}{*}{$\begin{array}{c}\text { Sinopharm (Wuhan) Unnamed }(2 \times 5 \\
\mu g \mathrm{vp}+\text { alum, } 21 \text { d.a. })[39]\end{array}$} & Post dose 1 & $112^{\mathrm{r}}$ & $14.3 \%$ & $6.0 \%$ & $0 \%$ & $0 \%$ & $3.6 \%$ & $1.2 \%$ \\
\hline & Post dose 2 & $112^{\mathrm{r}}$ & $10.7 \%$ & $9.5 \%$ & $0 \%$ & $0 \%$ & $0 \%$ & $1.2 \%$ \\
\hline
\end{tabular}


Table 2. Cont

\begin{tabular}{|c|c|c|c|c|c|c|c|c|}
\hline & \multirow[t]{2}{*}{ Dose } & \multirow[t]{2}{*}{$\mathbf{N}$} & \multicolumn{2}{|c|}{ Injection Site Pain } & \multicolumn{2}{|c|}{ Headache } & \multicolumn{2}{|c|}{ Fever } \\
\hline Grade & & & \multicolumn{2}{|c|}{ Any } & \multicolumn{2}{|c|}{ Any } & \multicolumn{2}{|c|}{ Oral $\geq 38^{\circ} \mathrm{C}$} \\
\hline \multirow{2}{*}{$\begin{array}{c}\text { Bharat Biotech COVAXIN }(2 \times 6 \mu \mathrm{g} \\
\text { Alhydroxiquim-II } 28 \text { d.a.) [42] }\end{array}$} & Post dose 1 & $175^{\mathrm{s}}$ & - & $3.2 \%$ & - & $0.5 \%$ & - & $4.2 \%$ \\
\hline & Post dose 2 & $175^{\mathrm{s}}$ & - & $2.6 \%$ & - & $1.5 \%$ & - & $2.1 \%$ \\
\hline
\end{tabular}

Notes: The most common solicited local and systemic reactogenicity were pain at the injection site, and headache, and these have been reported in Table 2 for 7 days following injection, except where noted. Reported also is fever defined as temperature $\geq 38{ }^{\circ} \mathrm{C}$ by oral route, except where noted. ${ }^{\mathrm{a}}$ 16-55 years and >55 years combined. ${ }^{\mathrm{b}}$ VRBPAC document, Tables 21 and 22 . November 25 dataset. ${ }^{\mathrm{c}}$ Ages $18-55$ y.o. No paracetamol. See Table S2 from reference [25]. ${ }^{\mathrm{d}}$ Ages 18+ y.o., mean of 3 age groups combined. See Table S11 from reference [27]. e See Table 2 from reference [28]. ${ }^{\mathrm{f}}$ The definition of fever was not provided in the publications. Severe fever was defined $>38.5{ }^{\circ} \mathrm{C}$ [29]. g Data reported for the liquid formulation using Ad26+Ad5 schedule from Table 2 in reference [30]. h Fever defined as $\geq 37.0{ }^{\circ} \mathrm{C}$, body location not indicated. ${ }^{j}$ Ages $\geq 18$ to $\leq 60$ years and $\geq 60$ years combined. Extracted from Figures 22 and 23 in reference [21]. $k$ Ages 18-59 years. Data extracted from Figure 2 in reference [34]. m SARS-CoV-2 seronegative adults (18-55 years). Supplementary Table S4 in reference [35]. P Adults aged 18-59 years. Reported within 14 days post first dose and 28 days post dose 2 . Phase 2 data from Tables A3-A6 in Appendix 2 in reference [36]. ${ }^{q}$ Adults aged 18-59 years. Fever not defined. Data are 7 days after 1 st or/and 2nd doses combined. Table 5 in reference [38]. ${ }^{\mathrm{r}}$ Adults aged 18-59 years. Fever defined as axillary temperature $>37.0^{\circ} \mathrm{C}$. Phase 2 data from Supplement 3 eTable 2 in reference [39]. ${ }^{s}$ Children and adults aged 12-64 years. Phase 2 data. Table 3 in reference [42].

Table 3 shows the immunogenicity data. A clear booster effect was observed after the second dose of both mRNA vaccines in both total anti-SARS-CoV-2 IgG and neutralization titers. There also appeared to be an increase in the proportion of spike-specific interferon (IFN) $-\gamma$ CD4 ${ }^{+} T$ cells after booster with the Moderna vaccine, but data are lacking for the post-first dose Pfizer vaccine at this time. Both mRNA vaccines showed evidence of a $\mathrm{T}$ helper (Th)1 bias.

\subsection{Viral Vector-Based (Non-Replicating) Vaccines-Safety/Reactogenicity and Immunogenicity/Efficacy}

AZ/Ox was first to release their published, peer-reviewed Phase III clinical trial data on 8 December 2020 based on an interim analysis of data out of sites in the UK, South Africa and Brazil [18]. Overall efficacy data based on 11,636 participants indicated a vaccine efficacy of $70.4 \%$. However, the waters were muddied somewhat when it was revealed that results were broken down into two different regimens. One subgroup of 2738 subjects received only half of the initial priming dose resulting in a $90 \%$ efficacy rate, while the larger group received the two standard doses of viral vector vaccine (Table 1), which resulted in a $62.1 \%$ efficacy rate. As for safety, in general, severe adverse events (SAEs) were distributed equally among placebo and vaccine groups, in contrast to the earlier safety trials [25], which indicated greater reactogenicity in the vaccine relative to the placebo group in terms of fever, pain, and headaches (Table 2). The viral vector vaccines presented in Table 2 resulted in fever in 16-18\% of subjects after the first dose. This is similar in frequency to the second dose of the mRNA vaccines, which was observed in $14-15.5 \%$ of subjects. Fever appeared to decrease after the second dose. In comparison, there was no increase in fever in Gardasil ${ }^{\circledR}-9$ recipients after the second and third doses [47].

The immunogenicity data from AZD1222 are promising [26] and appear to be comparable to those of the mRNA vaccines, although comparisons are difficult due to different assays employed (see Table 3 and accompanying notes).

Cansino's Ad5-nCoV (Convidecia) vaccine has been in use in China since mid-2020 well before Phase III trials were finished enrolling participants under a special 1-year military authorization (https://www.globaltimes.cn/content/1207517.shtml (accessed on 9 February 2021)). Press release information, released 8 February 2021, indicates a $65.7 \%$ vaccine efficacy for Convidecia, based on a study of 30,000 participants out of Pakistan (https: / / www.bloomberg.com/news / articles/2021-02-08/pakistan-says-cansinos-covid-vaccine-shows-65-7-efficacy (accessed on 9 February 2021)). The Phase II data in terms of safety, tolerability and immunogenicity [28,29] are adequate and indicative that (see Tables 2 and 3) the vaccine will be a useful addition to the COVID-19 vaccine armamentarium. 


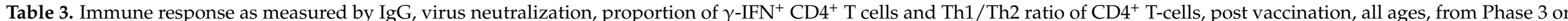
Phase $1 / 2$ using the final chosen formulation and schedule.

\begin{tabular}{|c|c|c|c|c|c|c|c|c|}
\hline & Timing & $\mathbf{N}$ & \multicolumn{2}{|c|}{ ELISA Titer } & \multicolumn{2}{|c|}{ Virus Neutralization } & $\gamma-\mathrm{IFN}^{+} \mathrm{CD}^{+} \mathrm{T}$ Cells & Th1/Th2 CD4 ${ }^{+} \mathrm{T}$ cells \\
\hline Units & $\begin{array}{c}\mathrm{dpd}=\text { Days Post } \\
\text { Dose }\end{array}$ & & \multicolumn{2}{|c|}{ GMT IgG } & \multicolumn{2}{|c|}{$\begin{array}{c}\text { GMT }=\text { Geometric Mean } \\
\text { Titer }\end{array}$} & $\%^{+}$ & Ratio of Measure \\
\hline Group & & $P+V$ & Placebo & Vaccine & Placebo & Vaccine & Vaccine & Vaccine \\
\hline \multicolumn{9}{|l|}{ mRNA } \\
\hline \multirow{2}{*}{$\begin{array}{c}\text { Pfizer/BioNTech } \\
\text { BNT162b2 } \\
(2 \times 30 \mu \mathrm{g}, 21 \text { d.a. })[16,43] \\
\end{array}$} & $21 \mathrm{dpd} 1$ & $\sim 30^{\mathrm{a}}$ & 0.8 & $665.7^{c}$ & 10.0 & $13.0^{\mathrm{d}}$ & - & - \\
\hline & $31 \mathrm{dpd} 2$ & $360^{\mathrm{b}}$ & 0.8 & $4931.7^{\mathrm{a}, \mathrm{c}}$ & 10.0 & $316.1^{\mathrm{d}}$ & $0.08 \% \mathrm{e}^{\mathrm{e}}$ & $4.4^{\mathrm{f}}$ \\
\hline \multirow{2}{*}{$\begin{array}{c}\text { Moderna } \\
\text { mRNA-1273 }(2 \times 100 \mu \mathrm{g}, 28 \text { d.a. })[22,44]\end{array}$} & 29 dpd 1 & $\sim 180^{\mathrm{g}}$ & 5.80 & 25.23 & 21.0 & $149.3^{\mathrm{h}}$ & $0.02 \%^{j}$ & $>>1^{\mathrm{j}}$ \\
\hline & 28 dpd 2 & $\sim 180^{g}$ & 5.86 & 147.42 & 21.2 & $1095.8^{\mathrm{h}}$ & $0.09 \%^{j}$ & $28.5^{\mathrm{j}}$ \\
\hline \multicolumn{9}{|l|}{ Viral Vector } \\
\hline \multirow{2}{*}{$\begin{array}{c}\text { Astra Zeneca AZD1222 } \\
\left(2 \times 5 \times 10^{10} \text { vp, } 28 \text { d.a. }\right)[18,25-27]\end{array}$} & 28 dpd 1 & 127 & $1^{\mathrm{k}}$ & $546^{\mathrm{k}}$ & $23.9 \mathrm{~m}$ & $203.2^{\mathrm{m}}$ & $0.06 \% \mathrm{p}$ & $6.3^{q}$ \\
\hline & 28 dpd 2 & 112 & - & $10,691^{\mathrm{n}}$ & - & $128^{\mathrm{n}}$ & $0.097 \%^{n}$ & - \\
\hline \multirow{2}{*}{$\begin{array}{c}\text { CanSino } \\
\text { Ad5-nCoV (single dose } 5 \times 10^{10} \text { vp) }[28,29]\end{array}$} & 28 dpd 1 & 255 & $20^{r}$ & $571^{\mathrm{r}}$ & $4^{\mathrm{s}}$ & $18.3^{\mathrm{s}}$ & $0.01 \%^{t}$ & - \\
\hline & NA & NA & NA & NA & NA & NA & NA & NA \\
\hline \multirow{2}{*}{$\begin{array}{l}\text { Gamaleya Gam-COVID-Vac } \\
\left(1 \times 10^{11} \text { vp Ad26 then Ad5 } 21 \text { d.a. }{ }^{a}\right)[19,30]\end{array}$} & $21 \mathrm{dpd} 1$ & 9 & $12.5^{\mathrm{u}}$ & $1721^{\mathrm{u}}$ & $1.25^{\mathrm{v}, \mathrm{w}}$ & $4.2^{\mathrm{v}}$ & $-^{x}$ & $-^{x}$ \\
\hline & $21 \mathrm{dpd} 2$ & 456 & - & $8996^{\mathrm{u}}$ & $1.6^{\mathrm{v}}$ & $44.5^{\mathrm{v}}$ & - & - \\
\hline $\begin{array}{l}\text { J\&J (Janssen) (Ad26.COV2.S } \\
\text { single dose } 5 \times 10^{10} \text { vp) [21,49] }\end{array}$ & 29 dpd 1 & $\sim 314$ & $<50$ & $399^{y}$ & $<58$ & $241^{z}$ & $0.09 \%^{\alpha}$ & $10^{\beta}$ \\
\hline \multicolumn{9}{|l|}{ Protein/VLP } \\
\hline \multirow{2}{*}{$\begin{array}{c}\text { Novavax } \\
\text { NVX-CoV2373 }(2 \times 5 \mu \mathrm{g}+50 \mu \mathrm{g} \text { M1, } 21 \text { d.a.) [34] }\end{array}$} & $21 \mathrm{dpd} 1$ & $\sim 52$ & $110^{\delta}$ & $1984^{\mathcal{\delta}}$ & $20^{\varepsilon}$ & $103^{\varepsilon}$ & - & - \\
\hline & 14 dpd 2 & $\sim 50$ & $114^{\delta}$ & $63,160^{\delta}$ & $20^{\varepsilon}$ & $3906^{\varepsilon}$ & $0.18 \%^{\zeta}$ & $7.5^{\zeta}$ \\
\hline \multirow{2}{*}{$\begin{array}{c}\text { Medicago } \\
\text { CoVLP }(2 \times 3.75 \mu \mathrm{g}+\text { AS03, } 21 \text { d.a. })[35]\end{array}$} & $21 \mathrm{dpd} 1$ & 20 & $64.8^{\mathrm{w}, \eta}$ & $4354^{\eta}$ & $5^{w, \theta}$ & $29.3^{\theta}$ & $0.01 \%^{\lambda}$ & $2.37^{\lambda}$ \\
\hline & 21 dpd 2 & 19 & - & $295,240^{\eta}$ & - & $811.3^{\theta}$ & $0.06 \%^{\lambda}$ & $1.4^{\lambda}$ \\
\hline \multicolumn{9}{|l|}{ Inactivated } \\
\hline \multirow{2}{*}{ Sinovac CoronaVac $(2 \times 3 \mu \mathrm{g}+$ alum, 14 d.a. $)[36,37]$} & 14 dpd 2 & $176^{\varepsilon}$ & 81 & 1094.3 & 2.0 & 27.6 & $0.00 \%^{\pi}$ & - \\
\hline & $28 \mathrm{dpd} 2$ & $176^{\varepsilon}$ & 80 & 1053.7 & 2.0 & 23.8 & $0.07 \%^{\pi}$ & - \\
\hline Units & $\begin{array}{c}\mathrm{dpd}=\text { Days Post } \\
\text { Dose }\end{array}$ & & \multicolumn{2}{|c|}{ GMT IgG } & \multicolumn{2}{|c|}{$\begin{array}{c}\text { GMT = Geometric Mean } \\
\text { Titer }\end{array}$} & $\%^{+}$ & Ratio of Measure \\
\hline \multirow{2}{*}{ Sinopharm (Beijing) BBIBP-CorV $2 \times 4 \mu \mathrm{g} v \mathrm{p}+$ alum, 21 d.a. [38] } & $14 \mathrm{dpd} 1^{\varsigma}$ & 112 & - & - & 2 & 219 & - & - \\
\hline & $28 \mathrm{dpd} 2 \varsigma$ & 112 & - & - & 2 & 282 & - & - \\
\hline \multirow{2}{*}{ Sinopharm (Wuhan) Unnamed $2 \times 5 \mu \mathrm{g} v \mathrm{p}+$ alum, 21 d.a. [39] } & $14 \mathrm{dpd} 1^{\sigma}$ & 56 & - & - & - & - & - & $0.92^{\varphi}$ \\
\hline & $14 \mathrm{dpd} 2^{\sigma}$ & 56 & 10 & 215 & 5 & 247 & & $1.21^{\varphi}$ \\
\hline
\end{tabular}


Table 3. Cont.

\begin{tabular}{|c|c|c|c|c|c|c|c|c|}
\hline & Timing & $\mathbf{N}$ & \multicolumn{2}{|c|}{ ELISA Titer } & \multicolumn{2}{|c|}{ Virus Neutralization } & $\gamma$-IFN ${ }^{+} \mathrm{CD}^{+}{ }^{+} \mathrm{T}$ Cells & Th1/Th2 CD4 ${ }^{+} \mathrm{T}$ cells \\
\hline Units & $\begin{array}{l}\text { dpd }=\text { Days Post } \\
\text { Dose }\end{array}$ & & \multicolumn{2}{|c|}{ GMT IgG } & \multicolumn{2}{|c|}{$\begin{array}{c}\text { GMT }=\text { Geometric Mean } \\
\text { Titer }\end{array}$} & $\%^{+}$ & Ratio of Measure \\
\hline \multirow{2}{*}{$\begin{array}{l}\text { Bharat Biotech COVAXIN }(2 \times 6 \mu \mathrm{\mu g} \text { in Alhydroxiquim-II adjuvant, } \\
\qquad 28 \text { d.a.) [40-42] }\end{array}$} & $28 \mathrm{dpd} 1 \psi$ & 190 & $500^{w}$ & 2240 & $5.6^{\mathrm{w}}$ & 12.8 & - & - \\
\hline & $28 \mathrm{dpd} 2 \psi$ & 190 & - & 9541 & - & 160 & - & $42 \omega$ \\
\hline
\end{tabular}

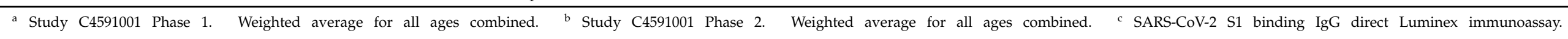

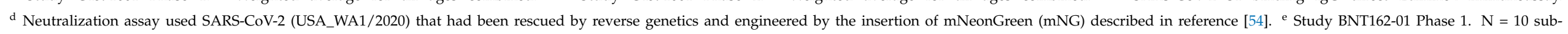

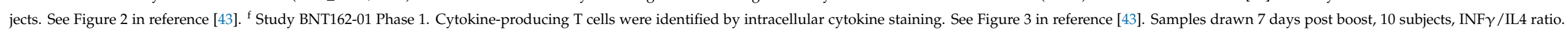

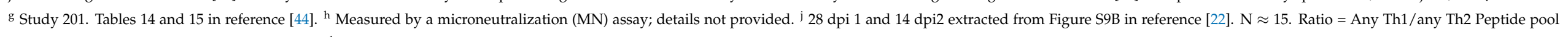

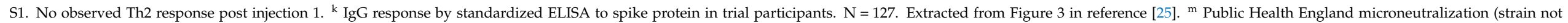

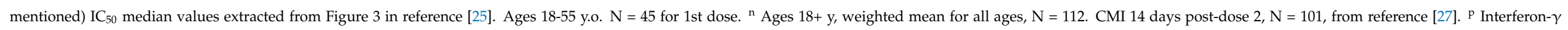

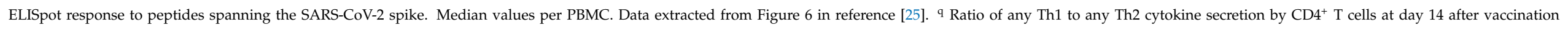

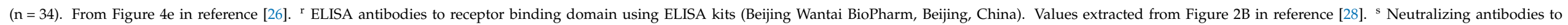

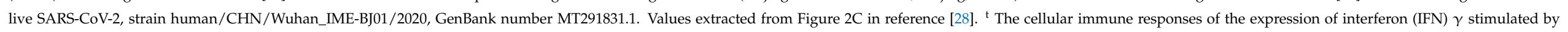

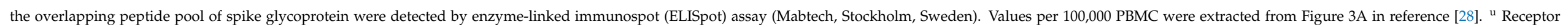

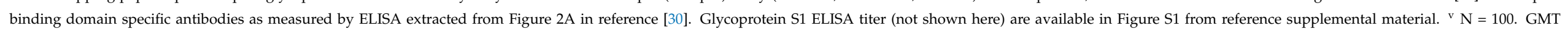

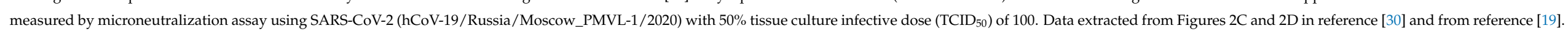

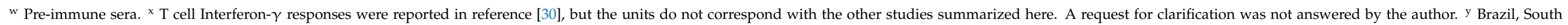

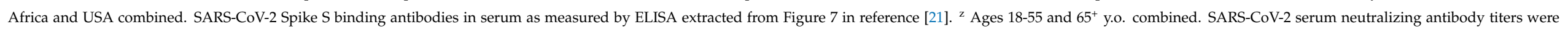

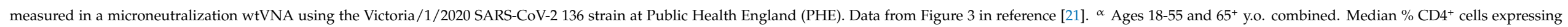

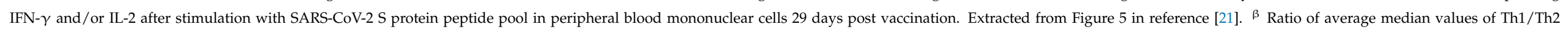

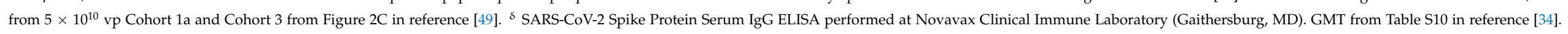

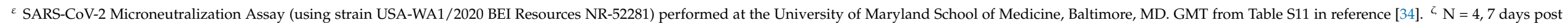

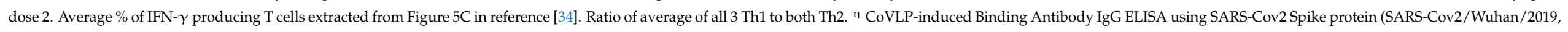

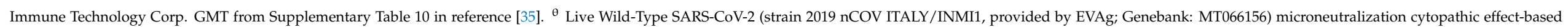

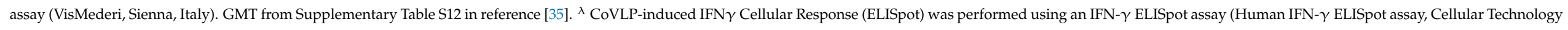

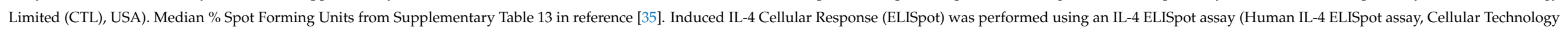

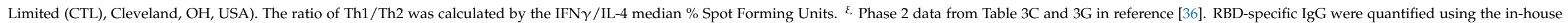

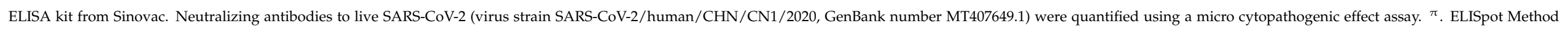

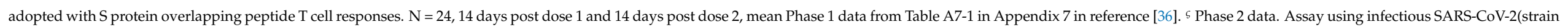

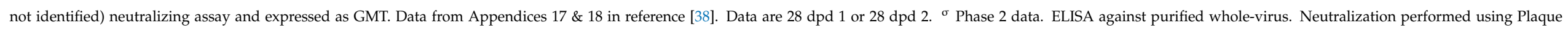

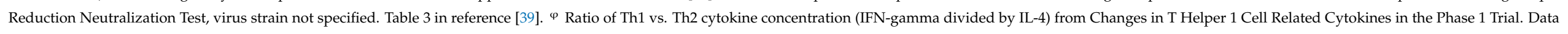

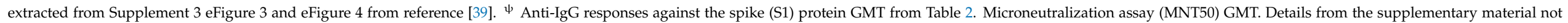

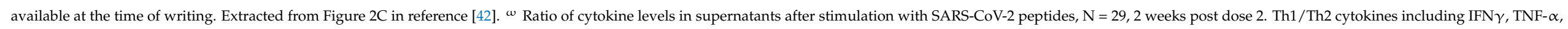
IL-2/IL-5, IL-10, IL-13. Extracted from Figure 4 in reference [42]. 
The Gamaleya Sputnik V Phase III data were released 2 February 2021 showing an efficacy rate of $91.6 \%$ based on an analysis of 78 COVID-19 cases placebo (62 cases) and vaccine (16 cases) in a 1:3 placebo/active vaccine randomized study (see Figure 1 and Table 1) [19,30]. Earlier published Phase I/II data [30] reveal an adequate safety profile but with elevated pain, fever, headaches in the vaccine group (Table 2) and the induction of a virus-specific antibody response (Table 3).

J\&J (Janssen) Ad26.COV2.S vaccine efficacy data from interim press release information (https: / / www.jnj.com/johnson-johnson-announces-single-shot-janssen-covid-19 -vaccine-candidate-met-primary-endpoints-in-interim-analysis-of-its-phase-3-ensemble-trial\#: :text=Phase $\% 203 \% 20$ ENSEMBLE $\% 20$ Study $\% 20$ Safety $\% 20$ Data\&text=Overall $\% 20 f e v$ er\%20rates $\% 20$ were $\% 209$, to $\% 20$ the $\% 20$ active $\% 20$ vaccine $\% 20$ candidate. (accessed on 9 February 2021)) indicate $66 \%$ efficacy across a range of countries where novel SARS-CoV-2 variants are emerging, such as in South Africa (see Figure 1, Table 1 and Section 5 below). Recently published Phase II data [31] and interim Phase III data provide evidence for acceptable safety, tolerability and immunogenicity (Tables 2 and 3).

\subsection{Protein-Based Vaccines-Safety/Reactogenicity and Immunogenicity/Efficacy}

Complete vaccine efficacy data are eagerly anticipated for this vaccine platform due to strong results pointing to excellent immunogenicity [34,35]. Interim data for the Novavax candidate NVX-CoV2373 points to 89.3\% efficacy (https: / / ir.novavax.com/news-releases / news-release-details / novavax-covid-19-vaccine-demonstrates-893-efficacy-uk-phase-3 (accessed on 9 February 2021)) (see Figure 1 and Table 1) and interesting facts are emerging related to vaccine efficacy against novel SARS-CoV-2 variants (see Section 5 below). This program has encountered significant delays in advancement, especially in the USA for a number of reasons including a high rate of placebo arm Phase III participants dropping out of trials to receive mRNA vaccines [55]. Medicago's plant-based protein/VLP candidate is lagging well behind in the field of twelve that initially announced Phase III trials.

Both protein/VLP candidate vaccines had an acceptable safety profile in Phase I/II trials, we know that both protein/VLP candidate vaccines are safe [34,35]. The primary adverse events include elevated pain at the injection site, and headache, primarily after the boosting dose (Table 2), which are common with all vaccines. An increase in percentage of subjects with fever was noted for the Medicago vaccine after the second dose, which was also observed for both mRNA vaccines (see Section 3.1). An increase in reactogenicity has been observed after re-vaccination with some vaccines, such as pertussis, varicella and tetanus, but the reasons for this are poorly understood [56].

Table 3 shows the immunogenicity data from earlier Phase II clinical trials. Both protein-based vaccines show strong increases in antiviral immune responses after the second dose. The Medicago vaccine increased antiviral antibody titer and spike protein specific IFN- $\gamma \mathrm{CD}^{+} \mathrm{T}$ cells after the booster dose, but only limited data were available for the Novavax vaccine at time of writing. Both vaccines show evidence for Th1 biased immune responses, attributed to the powerful adjuvants formulated with these vaccines [34,35].

\subsection{Inactivated Viral Vaccines - Safety/Reactogenicity and Efficacy/Immunogenicity}

There are some early indications that the inactivated viral vaccines possess lower efficacy compared to the mRNA platform vaccines but still with adequate protection marginally exceeding WHO guidelines of 50\% efficacy. The studies using Sinovac's CoronaVac vaccine have been overshadowed by discrepant figures cropping up in interim Phase III analyses from the different countries (ranging from a high of $91.25 \%$ in Turkey to a low of $65 \%$ in Indonesia) before finally settling on a $50.4 \%$ efficacy readout from nearly 10,000 subjects enrolled in Brazil. Sinopharm's (Beijing) candidate has a 79.3-86\% efficacy rate (the latter number coming from a study carried out in the UAE) with Sinopharm's (Wuhan) somewhat lower at 72.5\% (see Table 1). However, the full data sets are required for accurate assessment of efficacy for all three of the vaccines made in China. Efficacy of COVAXIN has not yet been reported as of February 2021 but it has been approved in India, 
so it is being assigned a $>50 \%$ efficacy readout (see Figure 1 and Table 1 ). As for safety, the incidence of pain, headache and fever was low from the inactivated virus platform and not significantly increased compared to placebo-injected recipients (Table 2). Adverse events were much lower than with mRNA or viral vector vaccines.

Some inactivated vaccines induce a Th2 immune response, which has been associated with vaccine associated enhanced respiratory disease (VAERD) in humans and animal models of coronavirus disease [57]. For this reason, there is of interest to know if the immune response induced by the different vaccine platforms are Th1 or Th2 biased [17,22,26,54]. Bharat Biotech's COVAXIN has used the novel adjuvant Algel-IMDG, which is an imidazoquinoline adsorbed on alum [42]. The Th1/Th2 ratio for this vaccine shows a very strong Th1 bias (Table 3) with a good reactogenicity profile (Table 2). The imidazoquinolines are Toll-like receptor $7 / 8$ agonists [58], and to the best of our knowledge, this is the first time it has been widely used as a vaccine adjuvant in humans.

\section{Comparative Analysis of Target Product Profiles}

Supplemental Table 1 lists the target product profile characteristics for a vaccine targeting COVID-19 from four sources. In this section, we focus specifically on five categories: (i) safety/reactogenicity; (ii) efficacy; (iii) dosing regimen; (iv) product stability/storage/supply chain (i.e., logistics); and (v) target price/accessibility (COGS). We start each category section with a "harmonized" TPP version of guidance from CEBR, CEPI, $\mathrm{WHO}$, and our previous publication. A ranking chart compares how well the vaccines match with the harmonized TPP using a scoring system of 1-5 and a weighting parameter for each category to provide a value out of 100 (Figure 2).

\section{Ranking according to Closeness to Target Product Profile Criteria}

\begin{tabular}{|c|c|c|c|c|c|c|}
\hline $\begin{array}{ll} & \text { Rank } \\
\text { ( ) Score }\end{array}$ & RNA & & Viral Vector & Protein & $\begin{array}{l}\text { Inactivat } \\
\text { Virus }\end{array}$ & \\
\hline $\begin{array}{l}\text { Safety/Reactogenicity } \\
\text { (Out of 30) }\end{array}$ & 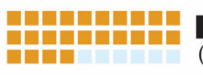 & $\begin{array}{l}4 \\
(24)\end{array}$ & $\begin{array}{c}\mathrm{x}=\mathrm{n} \\
\mathrm{(21)}\end{array}$ & 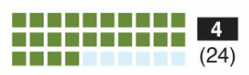 & 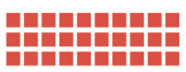 & $\begin{array}{c}5 \\
\\
(30)\end{array}$ \\
\hline Efficacy (50) & 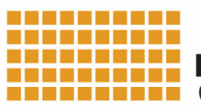 & $\frac{5}{(50)}$ & $\begin{array}{c}3.5 \\
\text { (35) }\end{array}$ & 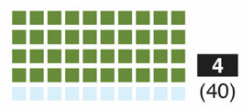 & gوtgen & $\begin{array}{l}2.5 \\
(25)\end{array}$ \\
\hline Logistics (10) & 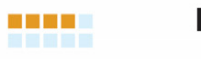 & $\frac{2}{(4)}$ & gen & gran & 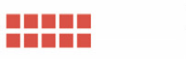 & $\frac{5}{(10)}$ \\
\hline COGS (10) & ㅁonn & $\frac{2}{(4)}$ & घng & 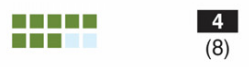 & gnen & $\frac{3}{(6)}$ \\
\hline $\begin{array}{l}\text { Total score } \\
(100)\end{array}$ & & & & & & \\
\hline
\end{tabular}

Notes: Ranking scale 1-5: 1 = does not satisfy, 2 = weakly satisfies, 3 = adequately satisfies, 4 = strongly satisfies, 5 = satisfies in all respects criteria of the harmonized TPP. Weighting factors, in parentheses) are $30,50,10,10$ for the respective categories.

Figure 2. Ranking of four vaccine platforms according to criteria for harmonized target product profile as mentioned in the text.

4.1. Safety/Reactogenicity-A SARS-CoV-2 Vaccine Should Provide a Clear Benefit/Risk Profile Based on $2^{+}$Data Sets with at Least 10,000 Subjects across All Age Groups with the Dosing Regimen Intended for Licensure

The minimum data sets and numbers of subjects enrolled and tested have clearly been met by the mRNA platform vaccines with young (16-18 y.o.) to elderly included. The first published datasets from the UK, Brazil, and South Africa Phase III AZ/Ox trials fell short in terms of numbers, especially with the altered dosing regimen [18] but this criteria will be met with additional data emerging [20], and when the numbers from the other three 
members of this platform are included. The same holds true for the four inactivated virus platform vaccine candidates, which have been tested in many countries (e.g., UAE, Bahrain, Indonesia, Turkey, China, India, etc.) and across demographics to meet the criteria of the harmonized TPP. With the recent release of interim data from the protein/VLP platform, with 15,000 enrolled in UK and 4000+ in South Africa this platform is near to meeting the harmonized TPP guide but would be strengthened by a dataset from Medicago's vaccine candidate.

In terms of safety/reactogenicity, the inactivated virus platform appears at the top (Ranking 5), while the others rank somewhat below. The viral vector platform scores below the other three platforms (Ranking 3.5) due to some lingering concerns related to paused trials and adverse events, more SAEs reported and the remote possibility for viral vector DNA integration into host cells (Figure 2).

4.2. Vaccine Efficacy-A SARS-CoV-2 Vaccine Should Demonstrate at Least $70 \%$ Efficacy, with Rapid Onset of Protection (<2 Weeks from Initial Dose), across All Age Groups, from Adolescents to Elderly with Appropriate Evaluation of Humoral and Cellular Immune Responses to Provide a Minimum Serological Correlate of Protection

The clear winners in this category are the mRNA vaccines (Ranking 5 ) with efficacy in the $95 \%$ range and across a spectrum of ages (from age 16 (Moderna) to elderly) and ethnicities, followed by the protein subunit platform (Ranking 4) with the recent data from Novavax revealed on 28 January 2021 with an efficacy of just under $90 \%$ in the UK. Next, is the viral vector platform (Ranking 3.5) with mixed efficacy between individual vaccine candidates in this group. The inactivated virus platform ranks lowest for this parameter (Ranking 2.5) based on currently available variable data from trials with Sinopharm and Sinovac vaccine candidates (Figure 2).

4.3. Compliance (Relating to Dosing Regimen) - A Sars-Cov-2 Vaccine Should Be a Single Dose during an Outbreak (Optimal). A Two-Dose Regimen Is Acceptable Provided There Is a Short Interval (<28 Days) between Prime and Boost (Minimal)

Since all but 2 of the 12 vaccines in the various platforms analyzed in this study use a two-dose regimen, all have been scored at the same ranking to meet the minimal harmonized TPP expectation for this parameter. Two of the four viral vector platform vaccines use a single dose (CanSino and J\&J/Janssen), which could argue for a ranking slightly above the others for this platform. However, since the scores are nearly identical, they are not included in the overall ranking depicted in Figure 2.

4.4. Logistics (Product Stability/Storage/Supply Chain)—A SARS-CoV-2 Vaccine Should Have a Shelf Life of at Least 1 Year at $-70^{\circ} \mathrm{C}$ and Be Stable for at Least 1 Month at Fridge Temperature $\left(4^{\circ} \mathrm{C}\right)$

Presumably, all platforms meet the harmonized TPP guideline for 1-year stability at $-70{ }^{\circ} \mathrm{C}$. However, there are considerable differences in vaccine stability at ordinary refrigeration temperatures between platforms with mRNA candidates possessing burdensome cold-chain requirements. This influences the logistics of vaccine storage and distribution and can lead to spoiled vaccines related to critical timing and handling issues. (Ranking 2). In contrast, the inactivated virus and viral vector platforms score highest (Ranking 5), with long-term stability at fridge temperature and relative ease of distribution. Stability information is not yet available for the protein-based vaccines, but these vaccines are formulated to make virus-like particles (VLPs). Similar VLPs include the Hepatitis B vaccine [59] and the AS03 adjuvanted influenza vaccine [60], both of which are stored at 2 to $8{ }^{\circ} \mathrm{C}$ long term. 
4.5. COGS (Target Price/Accessibility)—A SARS CoV-2 Vaccine Should Possess Capability for Rapid Scale-Up Production and Availability of Sufficient Doses at Cost/Dose That Allows Broad Use, Including in LMIC

While production of mRNA vaccines can be scaled-up at a reasonable pace, they are currently among the most expensive (https: / / s3.amazonaws.com/one.org/vaccine-accesstest/ONE_VAT_summary_November_EN.pdf (accessed on 9 February 2021)) COVID-19 vaccines, which diminishes their ranking in this category (Ranking 2). The inactivated virus vaccines are relatively easy to produce and are cheap if one considers Bharat Biotech's COVAXIN. However, there are some indications that the pricing of the vaccines made in China are very high (see three links in notes to Table 1) (Ranking 3). The viral vector vaccines are the cheapest to prepare (Ranking 5), with protein/VLP vaccines somewhat more expensive (Ranking 4). Manufacturing capacity and cost of goods (COGS) analyses give estimated values that match reasonably well with vaccine prices presented in Table 1 , affirming that most manufacturers are not making substantial profit on these vaccines during the pandemic. Manufacturing capacity also differs between platforms (Figure 3). Surprisingly, fill/finish is the bottleneck of the manufacturing platforms and contributes significantly to cost and time [61]. The Medicago protein-based platform matches the manufacturing capacity of mRNA vaccines ( 10 million doses per month), but each batch takes longer to make (Figure 3).

Vaccine Manufacturing compared

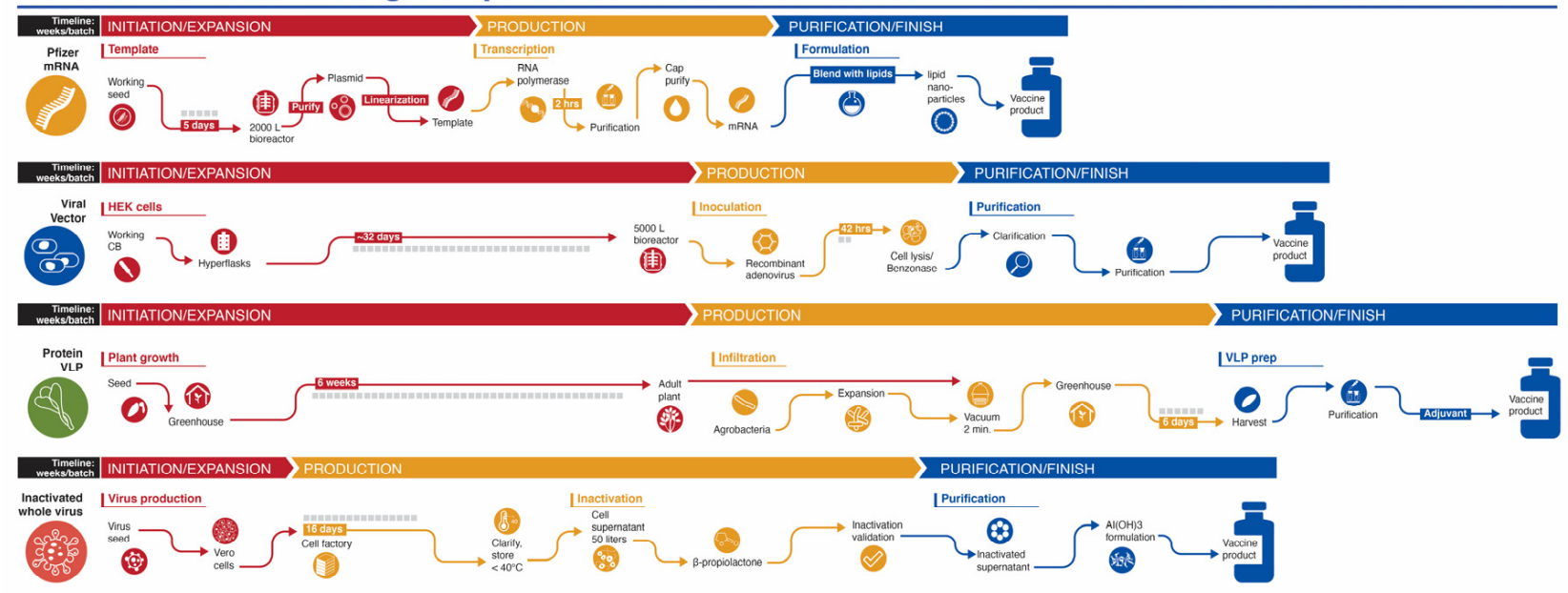

Figure 3. Manufacturing process of vaccines from four platforms covered in this review. The mRNA platform is represented by the Pfizer vaccine. The vaccine development process for the viral vector and inactivated whole virus platforms process are generic, while the protein/VLP platform is represented by the vaccine from Medicago. Timelines are not to scale.

\section{Discussion}

\subsection{Successes}

Firstly, the global concerted effort to bring a SARS-CoV-2 vaccine targeting COVID19 into the arms of millions of citizens around the world in less than a year has been a resounding triumph for science in general and for vaccine research and development in particular. The path has not been easy and there have been numerous challenges, obstacles, and significant suffering and death due to the virus worldwide. However, health care workers, front line essential workers, organizations, companies, and countries have rallied around the "global village" concept and the solidarity of cooperation that "we are all in this together" and "this won't end for anyone until it ends for everyone (https: / / www. nytimes.com/2020/04/07/opinion/coronavirus-united-states-leadership.html (accessed on 9 February 2021))". The successes of epidemiology, public health, genetic testing and diagnostics, rapid whole genome viral sequencing and outbreak surveillance should be lauded rather than sifting through the many shortcomings. 
Secondly, a novel vaccine platform (mRNA) has been established that will likely alter the course of vaccine development in perpetuity. This will pave the way for the use of the platform in cancer therapeutics and other biomedical applications.

\subsection{Barriers/Hurdles}

Firstly, while we know that antibody levels induced by natural infection with SARSCoV-2 last several months, we do not know if there will be a requirement for repeat vaccine dosing on an annual (or other timeframe) basis. If repeat booster doses are required, especially related to the viral vector platform, will antibodies be directed to the vector and will this diminish vaccine efficacy?

Secondly, determining an immunological correlate of protection against SARS-CoV-2 is an important objective that still has not been achieved and will be crucial in facilitating future COVID-19 vaccine development and licensing. Since the Pfizer and Moderna vaccines showed efficacy starting at 11 days after the first dose, the opportunity exists to identify a serological correlate of protection. The virus neutralization titer for the Pfizer vaccine was 13 after the first dose, just slightly above the cut-off for the assay, and 316 after the second dose. Likewise, the titer was 149 for the Moderna vaccine (first dose) and 1096 (second dose), respectively. A calculation based on early data shows a nearly significant correlation between vaccine efficacy versus post-vaccination neutralization $\log$-titer $\left(\mathrm{r}^{2}=0.39, p=0.07\right.$, see Supplementary Materials Table S3, Figure S1). It is possible that cell-mediated immunity (CMI) is a stronger correlate of protection against COVID-19 disease than antibody-mediated neutralization [62,63], but the failure to demonstrate a significant relationship may be simply due to the different methods used to measure neutralization titer. This calls for a global collaboration to develop standardized assays, as was done for the pneumococcal vaccine [64] and by the WHO for the meningococcal conjugate vaccine (https:/ / apps.who.int/iris/handle/10665/66298 (accessed on 9 February 2021)).

Thirdly, SARS-CoV-2 viral variants are emerging at an alarming rate from around the world [65-69]. Currently, the most discussed viral variants are those that have originated in the UK (known as B.1.1.7), South Africa (B.1.351; also known as 501Y.V2), and Brazil (P.1), respectively (see this resource for a discussion of these and others (https: / / www.the-scientist. $\mathrm{com} /$ news-opinion/a-guide-to-emerging-sars-cov-2-variants-68387?utm_campaign=TS_ DAILY_NEWSLETTER_2021\&utm_medium=email\&_hsmi=108329977\&_hsenc=p2ANqtz9N6VU4j0jsVdUrSG3a3WyIb9L1PLdJGrTihQesrURWRf6Dg143VX51\$ \times\$8TqqgidfNnTOuC1MqOBdMjHQD71SyUkL4UBvA\&utm_content=108329977\&utm_source=hs_email (accessed on 9 February 2021))). These variants each express a varied assortment of mutations (compared to the original SARS-CoV-2 Wuhan isolates) but all possess the asparagine $\rightarrow$ tyrosine N501Y mutation that appears to increase the ability of ACE2 to interact with the viral spike receptor-binding domain (RBD). Early indications suggest that the mRNA vaccines are still effective against B.1.1.7 [70-72]. Another mutation within the spike protein of variant B.1.351, glutamic acid $\rightarrow$ lysine E484K, is particularly troubling and this has perhaps led to the reduced efficacy of vaccination in Phase III clinical trials that have taken place in South Africa with the J\&J and Novavax vaccines (see notes d,e in the Table 1 legend). Currently, there is no direct evidence that the variants heighten disease severity but this important point is being investigated. These and other emerging variants possess the potential to derail vaccine efforts to eradicate SARS-CoV-2 viral spread. Moderna has already pre-emptively started production of a new mRNA vaccine candidate targeting B.1.351 and intends to carry out booster vaccinations with this new vaccine [68].

Finally, public perception and compliance in vaccine administration are also very large hurdles to surmount in order to achieve herd immunity in some countries/populations. While not discussed in this review, this is a key point that cannot be overlooked.

\subsection{Limitations of This Analysis}

To date only a few of the twelve vaccine candidates that had announced Phase III trials by mid-November 2020 have published their complete datasets from these trials. 
Some parts of the analyses have had to rely on partial or incomplete datasets for the Phase III trials or to make use of the Phase II data for some parameters. The rankings presented in Figure 2 are an approximation to the ideal SARS CoV-2 vaccine target product profile with not all fourteen specific criteria evaluated as listed in Supplemental Table S1.

\subsection{Concluding Remarks}

Ten of the twelve vaccines discussed in this article have received some form of authorization for use in different countries around the world as of the end of February 2021. Vaccine rollout has begun in massive campaigns, in Israel, UAE, and across the United States, while the process has been slow throughout Europe, Canada and developing nations. Early success is emerging in Israel [73] where approximately half the population has been vaccinated, with cases of COVID and hospitalization falling dramatically. There has been widespread concern of a two-tier system where rich nations monopolize the expensive, "high-tech" rapidly deployed mRNA vaccines, while other low and middle income countries (LMIC) receive the less-tested (and as-of-yet) incompletely revealed vaccine efficacy candidates. Nonetheless, the successes should be praised and hope should reign that the global pandemic will end soon or at least be held in check so that we can return to a world unhampered by restrictions and to global prosperity.

Supplementary Materials: The following are available online at https:/ /www.mdpi.com/1999-4 915/13/3/418/s1, Figure S1: Clinical efficacy vs neutralization titer (NT) for each vaccine with available data, Table S1: Target product profiles for COVID-19 vaccines, Table S2: Comparison of the formulation and stability of two mRNA vaccine lipid nanoparticles using information from the product monographs, Table S3: Correlation of vaccine clinical efficacy vs post-dose 2 virus neutralization GMT.

Author Contributions: C.D.F., C.L. and A.A. conceived the content and wrote the article. All authors have read and agreed to the published version of the manuscript.

Funding: No specific funding was directed towards this article.

Institutional Review Board Statement: Not applicable.

Informed Consent Statement: Not applicable. No human subjects were recruited as part of this study.

Data Availability Statement: All data are contained within the References section and Supplemental Materials.

Acknowledgments: We thank Ian Dennis for preparation of figures and Jennifer Cross for critical reading of the manuscript.

Conflicts of Interest: Author A.A. is founder and managing director of Novateur Ventures, Inc. C.D.K. and C.L. are consultants with Novateur Ventures Inc. The authors have no associations with the makers of the twelve vaccine candidates presented here.

\footnotetext{
Abbreviations

COVID-19: Coronavirus disease 2019; SARS, Severe Acute Respiratory Syndrome; Ab, antibody; Ad, adenovirus; AZ/Ox, Astra Zeneca/University of Oxford; TPP, target product profile; CEPI, Coalition for Epidemic Preparedness Innovations; dpd, days post-dose; GAVI, GAVI The Vaccine Alliance (formerly Global Alliance for Vaccines and Immunization); WHO, World Health Organization; FDA, United States Food and Drug Administration; EUA, emergency use authorization; ACTIV, Accelerating COVID-19 Therapeutic Interventions and Vaccines; VAERD, vaccine-associated enhanced respiratory disease; LNP, lipid nanoparticle; IFN, interferon; J\&J, Johnson \& Johnson/Janssen; VLP, virus-like particle; $\mathrm{AE}$, adverse event; $\mathrm{SAE}$, serious adverse event; Th, T helper; $\mathrm{CMI}$, cell-mediated immunity; COGS, cost of goods sold; LMIC, low-middle income countries; HPV, human papilloma virus; i.m., intramuscular; vp, viral particles; y.o., years old; VRBPAC, Vaccines and Related Biological Products Advisory Committee.
} 


\section{References}

1. Ahn, D.G.; Shin, H.J.; Kim, M.H.; Lee, S.; Kim, H.S.; Myoung, J.; Kim, B.T.; Kim, S.J. Current Status of Epidemiology, Diagnosis, Therapeutics, and Vaccines for Novel Coronavirus Disease 2019 (COVID-19). J. Microbiol. Biotechnol. 2020, 30, 313-324. [CrossRef]

2. Amanat, F.; Krammer, F. SARS-CoV-2 Vaccines: Status Report. Immunity 2020, 52, 583-589. [CrossRef]

3. Callaway, E. Scores of coronavirus vaccines are in competition-how will scientists choose the best? Nature 2020. [CrossRef]

4. Chen, G.; Wu, D.; Guo, W.; Cao, Y.; Huang, D.; Wang, H.; Wang, T.; Zhang, X.; Chen, H.; Yu, H.; et al. Clinical and immunological features of severe and moderate coronavirus disease 2019. J. Clin. Investig. 2020, 130, 2620-2629. [CrossRef]

5. Cohen, J. Vaccine designers take first shots at COVID-19. Science 2020, 368, 14-16. [CrossRef]

6. Cyranoski, D. Profile of a killer: The complex biology powering the coronavirus pandemic. Nature 2020, 581, 22-26. [CrossRef]

7. Du, Y.; Tu, L.; Zhu, P.; Mu, M.; Wang, R.; Yang, P.; Wang, X.; Hu, C.; Ping, R.; Hu, P.; et al. Clinical Features of 85 Fatal Cases of COVID-19 from Wuhan. A Retrospective Observational Study. Am. J. Respir. Crit. Care Med. 2020, 201, 1372-1379. [CrossRef]

8. Graham, B.S. Rapid COVID-19 vaccine development. Science 2020, 368, 945-946. [CrossRef] [PubMed]

9. Guan, W.-J.; Ni, Z.-Y.; Hu, Y.; Liang, W.-H.; Ou, C.-Q.; He, J.-X.; Liu, L.; Shan, H.; Lei, C.-L.; Hui, D.S.C.; et al. Clinical Characteristics of Coronavirus Disease 2019 in China. N. Engl. J. Med. 2020, 382, 1708-1720. [CrossRef]

10. Oberfeld, B.; Achanta, A.; Carpenter, K.; Chen, P.; Gilette, N.M.; Langat, P.; Said, J.T.; Schiff, A.E.; Zhou, A.S.; Barczak, A.K.; et al. SnapShot: COVID-19. Cell 2020, 181, 954. [CrossRef]

11. Le, T.T.; Andreadakis, Z.; Kumar, A.; Román, G.R.; Tollefsen, S.; Saville, M.; Mayhew, S. The COVID-19 vaccine development landscape. Nat. Rev. Drug Discov. 2020, 19, 305-306. [CrossRef] [PubMed]

12. Wu, F.; Zhao, S.; Yu, B.; Chen, Y.M.; Wang, W.; Song, Z.G.; Hu, Y.; Tao, Z.W.; Tian, J.H.; Pei, Y.Y.; et al. A new coronavirus associated with human respiratory disease in China. Nature 2020, 579, 265-269. [CrossRef] [PubMed]

13. Zhou, P.; Yang, X.L.; Wang, X.G.; Hu, B.; Zhang, L.; Zhang, W.; Si, H.R.; Zhu, Y.; Li, B.; Huang, C.L.; et al. A pneumonia outbreak associated with a new coronavirus of probable bat origin. Nature 2020, 579, 270-273. [CrossRef] [PubMed]

14. Zhu, N.; Zhang, D.; Wang, W.; Li, X.; Yang, B.; Song, J.; Zhao, X.; Huang, B.; Shi, W.; Lu, R.; et al. A Novel Coronavirus from Patients with Pneumonia in China, 2019. N. Engl. J. Med. 2020, 382, 727-733. [CrossRef] [PubMed]

15. Funk, C.D.; Laferrière, C.; Ardakani, A. A Snapshot of the Global Race for Vaccines Targeting SARS-CoV-2 and the COVID-19 Pandemic. Front. Pharmacol. 2020, 11, 937. [CrossRef] [PubMed]

16. Polack, F.P.; Thomas, S.J.; Kitchin, N.; Absalon, J.; Gurtman, A.; Lockhart, S.; Perez, J.L.; Pérez Marc, G.; Moreira, E.D.; Zerbini, C.; et al. Safety and Efficacy of the BNT162b2 mRNA Covid-19 Vaccine. N. Engl. J. Med. 2020, 383, 2603-2615. [CrossRef]

17. Baden, L.R.; El Sahly, H.M.; Essink, B.; Kotloff, K.; Frey, S.; Novak, R.; Diemert, D.; Spector, S.A.; Rouphael, N.; Creech, C.B. Efficacy and safety of the mRNA-1273 SARS-CoV-2 vaccine. N. Engl. J. Med. 2021, 384, 403-416. [CrossRef] [PubMed]

18. Voysey, M.; Clemens, S.A.C.; Madhi, S.A.; Weckx, L.Y.; Folegatti, P.M.; Aley, P.K.; Angus, B.; Baillie, V.L.; Barnabas, S.L.; Bhorat, Q.E.; et al. Safety and efficacy of the ChAdOx1 nCoV-19 vaccine (AZD1222) against SARS-CoV-2: An interim analysis of four randomised controlled trials in Brazil, South Africa, and the UK. Lancet 2021, 397, 99-111. [CrossRef]

19. Logunov, D.Y.; Dolzhikova, I.V.; Zubkova, O.V.; Tukhvatullin, A.I.; Shcheblyakov, D.V.; Dzharullaeva, A.S.; Grousova, D.M.; Erokhova, A.S.; Kovyrshina, A.V.; Botikov, A.G. Safety and efficacy of an rAd26 and rAd5 vector-based heterologous prime-boost COVID-19 vaccine: An interim analysis of a randomised controlled phase 3 trial in Russia. Lancet 2021, 397, P671-P681. [CrossRef]

20. Voysey, M.; Costa Clemens, S.A.; Madhi, S.A.; Weckx, L.Y.; Folegatti, P.M.; Aley, P.K.; Angus, B.J.; Baillie, V.; Barnabas, S.L.; Bhorat, Q.E. Single Dose Administration, and the Influence of the Timing of the Booster Dose on Immunogenicity and Efficacy Of ChAdOx1 nCoV-19 (AZD1222) Vaccine. Lancet 2021. Available online: https:/ /ssrn.com/abstract=3777268 (accessed on 9 February 2021). [CrossRef]

21. Janssen. COVID-19 Vaccine Ad26.COV2.S VAC31518 (JNJ-78436735) Sponsor Briefing Document Vaccines and Related Biological Products Advisory Committee 26 February 2021. Available online: https:/ /www.fda.gov/media/146219/download (accessed on 26 February 2021).

22. Jackson, L.A.; Anderson, E.J.; Rouphael, N.G.; Roberts, P.C.; Makhene, M.; Coler, R.N.; McCullough, M.P.; Chappell, J.D.; Denison, M.R.; Stevens, L.J.; et al. An mRNA Vaccine against SARS-CoV-2-Preliminary Report. N. Engl. J. Med. 2020, 383, 1920-1931. [CrossRef] [PubMed]

23. Walsh, E.E.; Frenck, R.W., Jr.; Falsey, A.R.; Kitchin, N.; Absalon, J.; Gurtman, A.; Lockhart, S.; Neuzil, K.; Mulligan, M.J.; Bailey, R. Safety and immunogenicity of two RNA-based Covid-19 vaccine candidates. N. Engl. J. Med. 2020, 383, 2439-2450. [CrossRef] [PubMed]

24. Editorial. Messengers of hope. Nat. Biotechnol. 2021, 39, 1. [CrossRef] [PubMed]

25. Folegatti, P.M.; Ewer, K.J.; Aley, P.K.; Angus, B.; Becker, S.; Belij-Rammerstorfer, S.; Bellamy, D.; Bibi, S.; Bittaye, M.; Clutterbuck, E.A.; et al. Safety and immunogenicity of the ChAdOx1 nCoV-19 vaccine against SARS-CoV-2: A preliminary report of a phase 1/2, single-blind, randomised controlled trial. Lancet 2020, 396, 467-478. [CrossRef]

26. Ewer, K.J.; Barrett, J.R.; Belij-Rammerstorfer, S.; Sharpe, H.; Makinson, R.; Morter, R.; Flaxman, A.; Wright, D.; Bellamy, D.; Bittaye, M.; et al. T cell and antibody responses induced by a single dose of ChAdOx1 nCoV-19 (AZD1222) vaccine in a phase $1 / 2$ clinical trial. Nat. Med. 2021, 27, 270-278. [CrossRef] [PubMed]

27. Ramasamy, M.N.; Minassian, A.M.; Ewer, K.J.; Flaxman, A.L.; Folegatti, P.M.; Owens, D.R.; Voysey, M.; Aley, P.K.; Angus, B.; Babbage, G.; et al. Safety and immunogenicity of ChAdOx1 nCoV-19 vaccine administered in a prime-boost regimen in young and old adults (COV002): A single-blind, randomised, controlled, phase 2/3 trial. Lancet 2021, 396, 1979-1993. [CrossRef] 
28. Zhu, F.C.; Guan, X.H.; Li, Y.H.; Huang, J.Y.; Jiang, T.; Hou, L.H.; Li, J.X.; Yang, B.F.; Wang, L.; Wang, W.J.; et al. Immunogenicity and safety of a recombinant adenovirus type-5-vectored COVID-19 vaccine in healthy adults aged 18 years or older: A randomised, double-blind, placebo-controlled, phase 2 trial. Lancet 2020, 396, 479-488. [CrossRef]

29. Zhu, F.C.; Li, Y.H.; Guan, X.H.; Hou, L.H.; Wang, W.J.; Li, J.X.; Wu, S.P.; Wang, B.S.; Wang, Z.; Wang, L.; et al. Safety, tolerability, and immunogenicity of a recombinant adenovirus type- 5 vectored COVID-19 vaccine: A dose-escalation, open-label, non-randomised, first-in-human trial. Lancet 2020, 395, 1845-1854. [CrossRef]

30. Logunov, D.Y.; Dolzhikova, I.V.; Zubkova, O.V.; Tukhvatullin, A.I.; Shcheblyakov, D.V.; Dzharullaeva, A.S.; Grousova, D.M.; Erokhova, A.S.; Kovyrshina, A.V.; Botikov, A.G.; et al. Safety and immunogenicity of an rAd26 and rAd5 vector-based heterologous prime-boost COVID-19 vaccine in two formulations: Two open, non-randomised phase $1 / 2$ studies from Russia. Lancet 2020, 396, 887-897. [CrossRef]

31. Sadoff, J.; Le Gars, M.; Shukarev, G.; Heerwegh, D.; Truyers, C.; de Groot, A.M.; Stoop, J.; Tete, S.; Van Damme, W.; Leroux-Roels, I.; et al. Interim Results of a Phase 1-2a Trial of Ad26.COV2.S Covid-19 Vaccine. N. Engl. J. Med. 2021. [CrossRef] [PubMed]

32. Knoll, M.D.; Wonodi, C. Oxford-AstraZeneca COVID-19 vaccine efficacy. Lancet 2021, 397, 72-74. [CrossRef]

33. Ledford, H. Oxford COVID-vaccine paper highlights lingering unknowns about results. Nature 2020, 588, 378-379. [CrossRef] [PubMed]

34. Keech, C.; Albert, G.; Cho, I.; Robertson, A.; Reed, P.; Neal, S.; Plested, J.S.; Zhu, M.; Cloney-Clark, S.; Zhou, H.; et al. Phase 1-2 Trial of a SARS-CoV-2 Recombinant Spike Protein Nanoparticle Vaccine. N. Engl. J. Med. 2020, 383, 2320-2332. [CrossRef]

35. Ward, B.J.; Gobeil, P.; Seguin, A.; Atkins, J.; Boulay, I.; Charbonneau, P.-Y.; Couture, M.; D'Aoust, M.-A.; Dhaliwall, J.; Finkle, C. Phase 1 trial of a Candidate Recombinant Virus-Like Particle Vaccine for Covid-19 Disease Produced in Plants. Medrxiv Prepr. Serv. Health Sci. 2020. Available online: https://www.medrxiv.org/content/10.1101/2020.11.04.20226282v1 (accessed on 9 February 2021).

36. Zhang, Y.; Zeng, G.; Pan, H.; Li, C.; Hu, Y.; Chu, K.; Han, W.; Chen, Z.; Tang, R.; Yin, W. Safety, tolerability, and immunogenicity of an inactivated SARS-CoV-2 vaccine in healthy adults aged 18-59 years: A randomised, double-blind, placebo-controlled, phase 1/2 clinical trial. Lancet Infect. Dis. 2021, 181-192. [CrossRef]

37. Palacios, R.; Patiño, E.G.; de Oliveira Piorelli, R.; Conde, M.; Batista, A.P.; Zeng, G.; Xin, Q.; Kallas, E.G.; Flores, J.; Ockenhouse, C.F.; et al. Double-Blind, Randomized, Placebo-Controlled Phase III Clinical Trial to Evaluate the Efficacy and Safety of treating Healthcare Professionals with the Adsorbed COVID-19 (Inactivated) Vaccine Manufactured by Sinovac-PROFISCOV: A structured summary of a study protocol for a randomised controlled trial. Trials 2020, 21, 853. [CrossRef]

38. Xia, S.; Zhang, Y.; Wang, Y.; Wang, H.; Yang, Y.; Gao, G.F.; Tan, W.; Wu, G.; Xu, M.; Lou, Z.; et al. Safety and immunogenicity of an inactivated SARS-CoV-2 vaccine, BBIBP-CorV: A randomised, double-blind, placebo-controlled, phase 1/2 trial. Lancet Infect. Dis. 2021, 21, 39-51. [CrossRef]

39. Xia, S.; Duan, K.; Zhang, Y.; Zhao, D.; Zhang, H.; Xie, Z.; Li, X.; Peng, C.; Zhang, Y.; Zhang, W.; et al. Effect of an Inactivated Vaccine Against SARS-CoV-2 on Safety and Immunogenicity Outcomes: Interim Analysis of 2 Randomized Clinical Trials. JAMA 2020, 324, 951-960. [CrossRef] [PubMed]

40. Ganneru, B.; Jogdand, H.; Daram, V.K.; Molugu, N.R.; Prasad, S.D.; Kannappa, S.V.; Ella, K.M.; Ravikrishnan, R.; Awasthi, A.; Jose, J. Evaluation of Safety and Immunogenicity of an Adjuvanted, TH-1 Skewed, Whole Virion Inactivated SARS-CoV-2 Vaccine-BBV152. iScience 2020. [CrossRef]

41. Ella, R.; Mohan, K.; Jogdand, H.; Prasad, S.; Reddy, S.; Sarangi, V.K.; Ganneru, B.; Sapkal, G.; Yadav, P.; Panda, S. Safety and immunogenicity trial of an inactivated SARS-CoV-2 vaccine-BBV152: A phase 1, double-blind, randomised control trial. Medrxiv Prepr. Serv. Health Sci. 2020. Available online: https://www.medrxiv.org/content/10.1101/2020.12.11.20210419v1 (accessed on 9 February 2021).

42. Ella, R.; Reddy, S.; Jogdand, H.; Sarangi, V.; Ganneru, B.; Prasad, S.; Das, D.; Raju, D.; Praturi, U.; Sapkal, G.; et al. Safety and immunogenicity clinical trial of an inactivated SARS-CoV-2 vaccine, BBV152 (a phase 2, double-blind, randomised controlled trial) and the persistence of immune responses from a phase 1 follow-up report. Medrxiv Prepr. Serv. Health Sci. 2020. [CrossRef]

43. Pfizer. Pfizer-Biontech COVID-19 Vaccine (BNT162, PF-07302048) Vaccines and Related Biological Products Advisory Committee Briefing Document. 2020. Available online: https:/ / www.fda.gov/media/144246/download (accessed on 9 February 2021).

44. Moderna. MRNA-1273 Vaccines and Related Biological Products Advisory Committee Meeting Presentation 17 December 2020. 2020. Available online: https:/ / www.fda.gov/media/144452/download (accessed on 9 February 2021).

45. Monograph. Prevnar®13, @ Wyeth Canada, 21 December 2009. 2009. Available online: https://pdf.hres.ca/dpd_pm/00052583 .PDF (accessed on 9 February 2021).

46. Monograph. SHINGRIX Herpes Zoster Vaccine (Non-Live Recombinant, AS01B Adjuvanted) 13 October 2017. 2017. Available online: https:/ / pdf.hres.ca/dpd_pm/00056533.PDF (accessed on 9 February 2021).

47. Monograph. GARDASIL®9 [Human Papillomavirus 9-Valent Vaccine, Recombinant] 5 February 2015. 2015. Available online: https:/ / pdf.hres.ca/dpd_pm/00058026.PDF (accessed on 9 February 2021).

48. Monograph. FLUVIRAL (2020-2021) Trivalent Influenza Vaccine (Split Virion, Inactivated) 6 February 2014. 2009. Available online: https:/ / pdf.hres.ca/dpd_pm/00057995.PDF (accessed on 9 February 2021).

49. Sadoff, J.; Le Gars, M.; Shukarev, G.; Heerwegh, D.; Truyers, C.; de Groot, A.M.; Stoop, J.; Tete, S.; Van Damme, W.; Leroux-Roels, I. Safety and immunogenicity of the Ad26. COV2. S COVID-19 vaccine candidate: Interim results of a phase 1/2a, double-blind, 
randomized, placebo-controlled trial. Medrxiv Prepr. Serv. Health Sci. 2020. Available online: https://www.medrxiv.org/content/ 10.1101/2020.09.23.20199604v1 (accessed on 26 February 2021).

50. Shimabukuro, T.T. Allergic reactions including anaphylaxis after receipt of the first dose of Pfizer-BioNTech COVID-19 vaccineUnited States, 14-23 December 2020. MMWR Morb. Mortal. Wkly. Rep. 2021, 70, 46-51. Available online: https://www.cdc.gov/ mmwr/volumes/70/wr/mm7002e1.htm (accessed on 9 February 2021).

51. Shimabukuro, T.T. Allergic Reactions including anaphylaxis after receipt of the first dose of Moderna COVID-19 vaccine-United States, 21 December 2020-10 January 2021. MMWR Morb. Mortal. Wkly. Rep. 2021, 70, 125-129. [CrossRef]

52. de Vrieze, J. Pfizer's vaccine raises allergy concerns. Science 2021, 371, 10-11. [CrossRef] [PubMed]

53. Szebeni, J. Complement activation-related pseudoallergy: A stress reaction in blood triggered by nanomedicines and biologicals. Mol. Immunol. 2014, 61, 163-173. [CrossRef] [PubMed]

54. Sahin, U.; Muik, A.; Derhovanessian, E.; Vogler, I.; Kranz, L.M.; Vormehr, M.; Baum, A.; Pascal, K.; Quandt, J.; Maurus, D.; et al. COVID-19 vaccine BNT162b1 elicits human antibody and T(H)1 T cell responses. Nature 2020, 586, 594-599. [CrossRef] [PubMed]

55. Dolgin, E. Search for better COVID vaccines confounded by existing rollouts. Nature 2021, 589, 340-341. [CrossRef]

56. Hervé, C.; Laupèze, B.; Del Giudice, G.; Didierlaurent, A.M.; Tavares Da Silva, F. The how's and what's of vaccine reactogenicity. NPJ Vaccines 2019, 4, 39. [CrossRef]

57. Bottazzi, M.E.; Strych, U.; Hotez, P.J.; Corry, D.B. Coronavirus vaccine-associated lung immunopathology-what is the significance? Microbes Infect. 2020, 22, 403-404. [CrossRef]

58. Vasilakos, J.P.; Tomai, M.A. The use of Toll-like receptor 7/8 agonists as vaccine adjuvants. Expert Rev. Vaccines 2013, 12, 809-819. [CrossRef] [PubMed]

59. Monograph. ENGERIX-B Hepatitis B Vaccine (Recombinant) 6 November 1987. 1987. Available online: https:// pdf.hres.ca/dpd_ pm/00058559.PDF (accessed on 9 February 2021).

60. Monograph. AREPANRIX ${ }^{\mathrm{TM}}$ H1N1 AS03-Adjuvanted H1N1 Pandemic Influenza Vaccine Emulsion for Injection, 20 April 2010. 2010. Available online: https://www.canada.ca/content/dam/hc-sc/migration/hc-sc/dhp-mps/alt_formats/pdf/ prodpharma/legislation/interimorders-arretesurgence/prodinfo-vaccin-eng.pdf (accessed on 9 February 2021).

61. Sedita, J.; Perrella, S.; Morio, M.; Berbari, M.; Hsu, J.S.; Saxon, E.; Jarrahian, C.; Rein-Weston, A.; Zehrung, D. Cost of goods sold and total cost of delivery for oral and parenteral vaccine packaging formats. Vaccine 2018, 36, 1700-1709. [CrossRef]

62. Sekine, T.; Perez-Potti, A.; Rivera-Ballesteros, O.; Strålin, K.; Gorin, J.B.; Olsson, A.; Llewellyn-Lacey, S.; Kamal, H.; Bogdanovic, G.; Muschiol, S.; et al. Robust T Cell Immunity in Convalescent Individuals with Asymptomatic or Mild COVID-19. Cell 2020, 183, 158-168. [CrossRef]

63. Wyllie, D.; Mulchandani, R.; Jones, H.E.; Taylor-Phillips, S.; Brooks, T.; Charlett, A.; Ades, A.E.; Makin, A.; Oliver, I.; Moore, P.; et al. SARS-CoV-2 responsive T cell numbers are associated with protection from COVID-19: A prospective cohort study in keyworkers. Medrxiv Prepr. Serv. Health Sci. 2020. [CrossRef]

64. Romero-Steiner, S.; Frasch, C.; Concepcion, N.; Goldblatt, D.; Käyhty, H.; Väkeväinen, M.; Laferriere, C.; Wauters, D.; Nahm, M.H.; Schinsky, M.F.; et al. Multilaboratory evaluation of a viability assay for measurement of opsonophagocytic antibodies specific to the capsular polysaccharides of Streptococcus pneumoniae. Clin. Diagn. Lab. Immunol. 2003, 10, 1019-1024. [CrossRef] [PubMed]

65. Kupferschmidt, K. Fast-spreading U.K. virus variant raises alarms. Science 2021, 371, 9-10. [CrossRef] [PubMed]

66. Kupferschmidt, K. Viral evolution may herald new pandemic phase. Science 2021, 371, 108-109. [CrossRef]

67. Kupferschmidt, K. Vaccinemakers ponder how to adapt to virus variants. Science 2021, 371, 448-449. [CrossRef] [PubMed]

68. Kupferschmidt, K. Vaccine 2.0: Moderna and Other Companies Plan Tweaks that Would Protect against New Coronavirus Mutations. Science 2021. [CrossRef]

69. Mahase, E. Covid-19: What new variants are emerging and how are they being investigated? BMJ 2021, 372, n158. [CrossRef]

70. Xie, X.; Zou, J.; Fontes-Garfias, C.R.; Xia, H.; Swanson, K.A.; Cutler, M.; Cooper, D.; Menachery, V.D.; Weaver, S.; Dormitzer, P.R.; et al. Neutralization of N501Y mutant SARS-CoV-2 by BNT162b2 vaccine-elicited sera. Biorxiv Prepr. Serv. Biol. 2021. [CrossRef]

71. Muik, A.; Wallisch, A.K.; Sänger, B.; Swanson, K.A.; Mühl, J.; Chen, W.; Cai, H.; Maurus, D.; Sarkar, R.; Türeci, Ö.; et al. Neutralization of SARS-CoV-2 lineage B.1.1.7 pseudovirus by BNT162b2 vaccine-elicited human sera. Science 2021. [CrossRef] [PubMed]

72. Xie, X.; Liu, Y.; Liu, J.; Zhang, X.; Zou, J.; Fontes-Garfias, C.R.; Xia, H.; Swanson, K.A.; Cutler, M.; Cooper, D.; et al. Neutralization of SARS-CoV-2 spike 69/70 deletion, E484K, and N501Y variants by BNT162b2 vaccine-elicited sera. Biorxiv Prepr. Serv. Biol. 2021. [CrossRef]

73. Mallapaty, S. Israel is first to see COVID-infection drop from vaccines. Nature 2021, 590, 197. [CrossRef] [PubMed] 